\title{
Bubble wall correlations in cosmological phase transitions
}

\author{
Ariel Mégevand $\odot^{*}$ and Federico Agustín Membiela ${ }^{\dagger}$ \\ IFIMAR (CONICET-UNMdP) Departamento de Física, Facultad de Ciencias Exactas y Naturales, \\ UNMdP, Deán Funes 3350, (7600) Mar del Plata, Argentina
}

(Received 12 August 2020; revised 28 September 2020; accepted 13 October 2020; published 12 November 2020)

\begin{abstract}
We study statistical relationships between bubble walls in cosmological first-order phase transitions. We consider the conditional and joint probabilities for different points on the walls to remain uncollided at given times. We use these results to discuss space and time correlations of bubble walls and their relevance for the consequences of the transition. In our statistical treatment, the kinematics of bubble nucleation and growth is characterized by the nucleation rate and the wall velocity as functions of time. We obtain general expressions in terms of these two quantities, and we consider several specific examples and applications.
\end{abstract}

DOI: 10.1103/PhysRevD.102.103514

\section{INTRODUCTION}

It is well known that first-order phase transitions may have occurred in the early Universe, and may have left several potentially observable remnants. In a cosmological first-order phase transition, a metastable high-temperature phase (false vacuum) undergoes supercooling, and then the phase transition proceeds through the nucleation and expansion of bubbles of the low-temperature stable phase (true vacuum). The dynamics is different in the case of a "vacuum" transition and in the case of a "thermal" transition [1,2]. In the former case, the nucleation of bubbles occurs in the absence of a plasma, and the nucleation rate $\Gamma$ is given by the probability of decay of the false vacuum per unit time per unit volume [3,4]. Besides, all the false-vacuum energy, which is released at the bubble walls, goes into accelerating the latter, which reach velocities $v \simeq 1$. This may also occur in a thermal phase transition with extreme supercooling, in which the wall velocity may exhibit runaway behavior [5-7]. The bubble walls disappear as bubbles collide, and the energy stored in the walls is transferred to thermal energy. On the other hand, a thermal transition occurs in the presence of a plasma, and we have a temperature-dependent nucleation rate $\Gamma(T)[8,9]$. In this case, the walls generally reach a terminal velocity $v(T)$, and most of the released energy (latent heat) goes to the fluid. Therefore, as the walls move, a reheating of the plasma occurs, as well as bulk fluid motions.

Even in the thin wall approximation, which is generally valid, the dynamics of thermal phase transitions is complex. In the first place, the nucleation rate is very sensitive to

\footnotetext{
*Member of CONICET, Argentina. megevand@mdp.edu.ar

Member of CONICET, Argentina. membiela@mdp.edu.ar
}

temperature variations. In the second place, the wall velocity depends on the microphysics, which determines the friction with the plasma $[10,11]$, and is also affected by the hydrodynamics [12-14]. Nevertheless, in many cases it is possible to assume that the nucleation rate is homogeneous and that the bubbles are spherical and all expand with the same speed. In such cases, the bubble kinematics is determined by the two basic ingredients $\Gamma(t)$ and $v(t)$. The kinematics is also affected, to a greater or lesser extent, by the scale factor $a(t)$. In most cases, however, the variation of the latter can be neglected for the duration of the phase transition. An exception is the case of strongly supercooled phase transitions $[1,15,16]$. In the statistical treatment of the phase transition, the quantities are averaged over possible realizations, and we shall use $\langle Q\rangle$ to denote the ensemble average of a quantity $Q$. In practice, these averages are calculated from the average number of bubbles nucleated in a given volume during a certain time, which is given by $\Gamma(t)$.

In the development of the transition, the most evident measure of progress is the volume fraction occupied by bubbles, $f_{b}(t)$. However, other quantities can be used as well, such as the fraction of bubble wall, which remains uncollided, $f_{S}(t)$ [1]. Since the collided walls quickly ${ }^{1}$ disappear inside merged bubbles, ${ }^{2}$ the uncollided wall area is essentially the total area $S_{\text {tot }}$ that is physically present at time $t$. We have $S_{\text {tot }}=\sum_{i} S_{i}$, where $S_{i}$ is the wall area of bubble $i$ which remains (uncollided) at time $t$. The fraction

\footnotetext{
${ }^{1}$ In general, all the terms in the equation of motion for the scalar field (order parameter) involve a single scale, namely, the scale of the theory, which is $\sim T$, so the characteristic timescale for the wall dynamics is $\sim T^{-1}$. This time is generally much shorter than the duration of the phase transition, which is determined by the Hubble rate and involves also the Planck scale.

${ }^{2}$ For recent discussions on the behavior of the scalar field after bubble collisions, see [17] and references therein.
} 
of surface $f_{S}$ is defined as $S_{\text {tot }}$ divided by the total area $\sum_{i} 4 \pi R_{i}^{2}$ of bubbles of radii $R_{i}$, including area that has been covered by bubbles. This quantity varies from $f_{S}=1$ at the beginning of the phase transition (when bubbles are isolated) to $f_{S}=0$ at the end (when all bubbles have merged and their walls have disappeared). The quantity $f_{S}$ tracks the conversion of potential energy (false vacuum energy or latent heat) to other forms of energy (kinetic energy of the wall, kinetic energy of the fluid, or thermal energy). It will be more relevant than $f_{b}$ to those processes involving the bubble walls. In particular, the departures from equilibrium which give rise to the important consequences of the phase transition originate at the bubble walls. Let us consider a few examples.

Baryogenesis.- If the electroweak phase transition is of first order, the walls of expanding bubbles push a net charge density into the symmetric phase, which bias baryonnumber violating processes [18-21]. This mechanism relies on diffusion processes that take place up to a distance $l$ from the wall, which is naturally $l \sim T^{-1}$. This length is several orders of magnitude smaller than the typical bubble radius $R$, which is of order $H^{-1}$, where $H$ is the Hubble rate. It is in this very thin shell next to the bubble walls where baryon number generation occurs. The baryon number density $n_{B}(t)$, which is left behind by the walls, depends on the value of the wall velocity. The latter is often estimated at the onset of nucleation, although $v(t)$ generally varies during the phase transition.

Gravitational waves.-The energy that is set in motion by the bubble walls is a source of gravitational waves (GWs) [22-24]. This energy may be concentrated in the walls themselves, or it may be transferred to bulk fluid motions $[2,25]$. In the latter case, the relation of the wall surface with the generated GW spectrum is indirect, since the direct source of GWs is the turbulence [2] or the sound waves [26] caused in the fluid, which may last longer than the phase transition. In any case, the walls are the source of such fluid motions. Furthermore, in the so-called bubblecollision mechanism, the energy transferred to the fluid is assumed to be concentrated in a thin shell around the walls. In the envelope approximation [27], the contribution of the overlap regions to the gravitational radiation is neglected, and the energy-momentum tensor is concentrated in the "envelope" of walls surrounding a cluster of bubbles.

Topological defects.-Perhaps the simplest example illustrating the formation of topological defects [28] is the trapping of a vortex in two spatial dimensions. Consider the spontaneous symmetry-breaking of a global $U(1)$ symmetry, where a complex scalar field $\phi$ vanishes in the symmetric phase and takes nonvanishing values $\phi=$ $v e^{i \alpha}$ in the broken-symmetry phase. The modulus $v$ is fixed but the phase $\alpha$ is arbitrary and uncorrelated in different bubbles. When two bubbles meet, $\alpha$ will rearrange itself so that it varies smoothly from one bubble to the other. Moreover, this phase will tend to take a constant value throughout space. When three bubbles meet at a given point, $\alpha$ will tend to vary smoothly along a closed line across the three bubbles. However, a complete equilibration to reach a constant phase may be topologically impossible, in which case a defect will be trapped in a symmetric-phase region enclosed by the bubbles. Nevertheless, it is very unlikely that three bubble walls collide simultaneously at a single point. Two of the bubbles will meet first, and the third one will arrive later. If the phase equilibration [29] between the first two bubbles completes before the arrival of the third bubble, the formation of a vortex may be avoided [30].

It is clear that the wall dynamics plays a relevant role in the determination of these consequences of the phase transition. In particular, electroweak baryogenesis takes place in a thin shell next to the bubble walls. Since the baryon number density depends on the wall velocity, a precise evaluation of the baryon asymmetry requires integrating $n_{B}(t)$ in time, weighted with the volume $\left\langle S_{\text {tot }}(t)\right\rangle v(t) d t$. In the case of GW production (at least in the envelope approximation), the energy-momentum tensor $T$ is also localized in a thin shell around the bubble walls. However, the spectrum of GWs depends on the correlation function $\langle T(x) T(y)\rangle$ between different space-time points (see, e.g., [31,32]). One would then expect that the GW spectrum will be related to bubble surface correlations $\left\langle S(t) S\left(t^{\prime}\right)\right\rangle$ rather than to the average area. On the other hand, for topological defect formation, the probability of trapping a defect in a sequence of bubble collisions depends on the probability that a point on a given bubble wall will soon collide once a nearby point has already collided. This mechanism should then be related to the correlation between different points on the same bubble wall.

It is well known that the probability that a random point of space is in the false vacuum is the same as the fraction of volume remaining in that phase. Also, the fraction of the bubble wall that remains uncollided is given by the probability that a point on a bubble wall remains in the false vacuum (this probability is not the same as the previous one, since the nucleation in the vicinity of the point is affected by the presence of the bubble to which it belongs [1]). Similarly, the surface correlations mentioned above will depend on conditional or joint probabilities for multiple points belonging to bubble walls.

In the present paper, we consider such probabilities. In the next section we review some existing results and discuss the probability that a set of arbitrary points in space remain in the false vacuum at a given time. In Sec. III we calculate the probability that a point in the surface of a bubble is uncollided, depending on whether another point in the same bubble wall or in the wall of another bubble is still uncollided. In Sec. IV we apply these results to discuss space and time correlations in the envelope of uncollided walls. We compare the results for different models. In Sec. V we consider some specific applications to the 
cosmological consequences of the transition. Finally, in Sec. VI, we summarize our conclusions.

\section{PHASE TRANSITION DYNAMICS AND PROBABILITY OF REMAINING IN THE FALSE VACUUM}

\section{A. Global dynamics}

As already mentioned, the relevant quantities in the kinematics of the phase transition are the nucleation rate $\Gamma$ and the wall velocity $v$. We shall assume that these quantities are homogeneous and given by certain functions of time $\Gamma(t), v(t)$. In particular, a homogeneous $v(t)$ implies that bubbles are spherical and that all expand with the same velocity. Although these assumptions are very common, they are not always valid. The nucleation rate is certainly homogeneous in the case of a vacuum transition, where $\Gamma$ is of the form $\Gamma=A e^{-S}$, with $A$ and $S$ constant $[3,4]$. Furthermore, in this case the wall velocity quickly approaches the asymptotic value $v=1$. In the case of a thermal transition, $\Gamma$ has a similar form, but $A$ and $S$ depend on the temperature $[8,9]$, and may thus have space and time variations. In general, the bubble walls reach a terminal velocity in a time that is much shorter than the total duration of the phase transition, but this velocity depends also on the temperature. In a phase transition mediated by detonations [33], the latent heat that is released at the bubble walls only reheats the plasma behind the walls (inside the bubbles), so $\Gamma$ and $v$ are not affected by temperature inhomogeneities. In contrast, for deflagrations [34-36], the fluid is perturbed in front of the bubble walls, and perturbations coming from different bubbles cause inhomogeneous reheating [14]. Even in this case, homogeneous $\Gamma$ and $v$ are often assumed to study the kinetics of the phase transition (see, e.g., [37]). Nevertheless, in the case of very slow deflagrations (with $v \lesssim 0.1$ ), the released latent heat is distributed with a relatively high speed, so a homogeneous reheating can be assumed [38].

In this section and the following we shall consider arbitrary functions $\Gamma(t)$ and $v(t)$, while in Secs. IV and $\mathrm{V}$ we shall consider particular examples. Actually, rather than the wall velocity, the basic ingredient here will be the bubble radius. Between two times $t^{\prime}$ and $t$, the radius of a bubble increases by

$$
R\left(t^{\prime}, t\right)=\int_{t^{\prime}}^{t} v\left(t^{\prime \prime}\right) d t^{\prime \prime} .
$$

For simplicity, we shall ignore the effect of the scale factor $a(t)$, which would introduce a factor $a(t) / a\left(t^{\prime \prime}\right)$ in the integrand. As already mentioned, this approximation is valid in most cases. In any case, generalizing our treatment to include this effect should be straightforward. If we neglect the initial radius of the bubble at the time of its nucleation, which is also a good approximation in general, then Eq. (1) gives the radius of a bubble that has nucleated at time $t^{\prime}$ and has expanded until time $t$. The fraction of volume occupied by bubbles is given by $f_{b}=1-P_{f v}$, where $P_{f v}$ is the fraction of volume in the false vacuum, which coincides with the probability that an arbitrary point is in that phase. This quantity is well known [39]. We shall consider a derivation here, which we shall generalize to less simple cases below.

\section{B. Probability that a given point in space remains in the false vacuum}

By time $t$, a point $p$ may have been reached by bubbles nucleated at times $t^{\prime \prime}<t$. We begin by considering the probability $P_{\text {out }}\left(t^{\prime \prime}\right)$ that $p$, at time $t$, is outside of any bubbles nucleated before a certain $t^{\prime \prime}$. This probability depends also on $t$, which we omit for simplicity of notation. Then, the probability that $p$ remains outside of any bubbles nucleated before $t^{\prime \prime}+d t^{\prime \prime}$ is given by the product

$$
P_{\text {out }}\left(t^{\prime \prime}+d t^{\prime \prime}\right)=P_{\text {out }}\left(t^{\prime \prime}\right)\left(1-d P\left(t^{\prime \prime}\right)\right),
$$

where the last factor is the probability that $p$ was not reached by bubbles nucleated between $t^{\prime \prime}$ and $t^{\prime \prime}+d t^{\prime \prime}$ either. That is to say, $d P\left(t^{\prime \prime}\right)$ is the probability that $p$ has been reached by some bubble nucleated between $t^{\prime \prime}$ and $t^{\prime \prime}+d t^{\prime \prime}$, assuming that $p$ was not reached by bubbles nucleated before $t^{\prime \prime}$. From Eq. (2) we readily obtain a differential equation whose solution is

$$
P_{\text {out }}\left(t^{\prime \prime}\right)=e^{-\int_{t_{c}}^{t^{\prime \prime}} d P} .
$$

Here, $t_{c}$ is the initial time, corresponding to the critical temperature $T_{c}$ of the phase transition, before which the nucleation rate vanishes. Evaluating at $t^{\prime \prime}=t$ we obtain the probability that the point $p$ remains in the false vacuum at time $t$,

$$
P_{f v}(t)=e^{-\int_{t_{c}}^{t} d P\left(t^{\prime \prime}\right)} .
$$

We still have to compute the conditional probability $d P\left(t^{\prime \prime}\right)$ that (at time $\left.t\right) p$ is inside a bubble nucleated between $t^{\prime \prime}$ and $t^{\prime \prime}+d t^{\prime \prime}$, assuming that it is outside of any previously nucleated bubbles. For a bubble nucleated at time $t^{\prime \prime}$ to reach the point $p$ before time $t$, the bubble must have nucleated at a distance smaller than $R\left(t^{\prime \prime}, t\right)$ from the point. In Fig. 1, the dots represent the possible nucleation points. To calculate the probability that a bubble was nucleated within this radius at time $t^{\prime \prime}$, we must determine whether, at that time, the whole region was actually available for bubble nucleation, since part of the space could have been occupied by previously nucleated bubbles. Nevertheless, such bubbles would also reach the point $p$ before time $t$, which we are assuming does not occur. Indeed, consider a bubble nucleated at a certain $t_{\text {prev }}<t^{\prime \prime}$. 


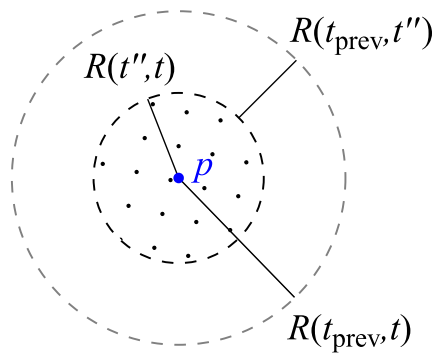

FIG. 1. The region in which bubbles must nucleate at time $t^{\prime \prime}$ in order to reach the point $p$ before time $t$ (dots). The outer circle indicates the region within which bubbles should nucleate at $t_{\text {prev }}<t^{\prime \prime}$ in order to affect the dotted region.

For this bubble to invade the dotted region at $t^{\prime \prime}$, it must have nucleated at a distance smaller than $R\left(t_{\text {prev }}, t^{\prime \prime}\right)$ from it (see Fig. 1). But then it would be too close to $p$, at a distance smaller than $R\left(t_{\text {prev }}, t^{\prime \prime}\right)+R\left(t^{\prime \prime}, t\right)=R\left(t_{\text {prev }}, t\right)$.

Hence, the whole volume of the dotted region is free of bubbles at time $t^{\prime \prime}$ and is available for nucleations. Thus, the probability $d P\left(t^{\prime \prime}\right)$ is given by

$$
d P\left(t^{\prime \prime}\right)=d t^{\prime \prime} \Gamma\left(t^{\prime \prime}\right) \frac{4 \pi}{3} R\left(t^{\prime \prime}, t\right)^{3}
$$

From (4)-(5) we obtain

$$
P_{f v}(t)=e^{-I(t)},
$$

where

$$
I(t)=\int_{t_{c}}^{t} d t^{\prime \prime} \Gamma\left(t^{\prime \prime}\right) \frac{4 \pi}{3} R\left(t^{\prime \prime}, t\right)^{3}
$$

\section{Probability that a point is in the false vacuum given that another point is in the false vacuum}

Let us now consider the probability $P_{p \mid p^{\prime}}$ that a point $p$ remains in the false vacuum at time $t$, given that another point $p^{\prime}$ was in the false vacuum at time $t^{\prime} \leq t$. Proceeding as before, we consider the probability $P_{\text {out }}\left(t^{\prime \prime}\right)$ that $p$ has not been reached by bubbles nucleated before $t^{\prime \prime}$, and then the probability $d P\left(t^{\prime \prime}\right)$ that $p$ has been reached by a bubble nucleated between $t^{\prime \prime}$ and $t^{\prime \prime}+d t^{\prime \prime}$. Thus, we obtain the same equation for $P_{\text {out }}\left(t^{\prime \prime}\right)$, Eq. (2), which leads to Eqs. (3) and (4). Like in the previous case, $d P\left(t^{\prime \prime}\right)$ is the conditional probability that $p$ is inside a bubble nucleated between $t^{\prime \prime}$ and $t^{\prime \prime}+d t^{\prime \prime}$ subjected to the condition that $p$ is outside of bubbles nucleated before $t^{\prime \prime}$. The difference is that, in the present case, we also have the condition that the other point, $p^{\prime}$, is in the false vacuum at time $t^{\prime}$. Therefore, we write

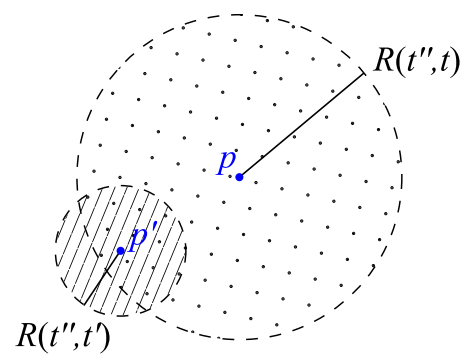

FIG. 2. Regions affecting the points $p$ and $p^{\prime}$ for $t^{\prime \prime}<t^{\prime}<t$. The dotted region is that in which a bubble must nucleate at time $t^{\prime \prime}$ in order to reach the point $p$ before time $t$. If the nucleation occurs inside the striped region, the bubble would eat the point $p^{\prime}$ before time $t^{\prime}$.

$$
P_{p \mid p^{\prime}}=e^{-\int_{t_{c}}^{t} d P\left(t^{\prime \prime}\right)},
$$

and we must reevaluate the conditional probability $d P\left(t^{\prime \prime}\right)$.

At $t^{\prime \prime}$, the bubble affecting $p$ must have nucleated within a sphere of radius $R\left(t^{\prime \prime}, t\right)$ centered at this point, like in Fig. 1. Then, in principle, we would obtain Eq. (5). Again, under the present conditions the dotted region is not affected, at time $t^{\prime \prime}$, by previously nucleated bubbles. However, the nucleations at $t^{\prime \prime}$ might reach the point $p^{\prime}$ before time $t^{\prime}$, which is now forbidden by the conditional probability. For $t^{\prime \prime}>t^{\prime}$ this will not happen, so we still have

$$
d P\left(t^{\prime \prime}\right)=d t^{\prime \prime} \Gamma\left(t^{\prime \prime}\right) \frac{4 \pi}{3} R\left(t^{\prime \prime}, t\right)^{3}, \quad\left(t^{\prime \prime}>t^{\prime}\right) .
$$

But for $t^{\prime \prime} \leq t^{\prime}$, any nucleation at time $t^{\prime \prime}$ must occur at a distance larger than $R\left(t^{\prime \prime}, t^{\prime}\right)$ from $p^{\prime}$ in order to avoid affecting this point. This situation is represented in Fig. 2. A nucleation at $t^{\prime \prime}$ must occur inside the dotted region in order to affect the point $p$ but outside the striped region to leave $p^{\prime}$ unaffected. Therefore, we have

$d P\left(t^{\prime \prime}\right)=d t^{\prime \prime} \Gamma\left(t^{\prime \prime}\right)\left[\frac{4 \pi}{3} R\left(t^{\prime \prime}, t\right)^{3}-V_{\cap}\right], \quad\left(t^{\prime \prime} \leq t^{\prime}\right)$,

where $V_{\cap}$ is the volume of the intersection of the two spheres.

From Eqs. (8)-(10), we obtain

$P_{p \mid p^{\prime}}=\exp \left[-\int_{t_{c}}^{t} d t^{\prime \prime} \Gamma\left(t^{\prime \prime}\right) \frac{4 \pi}{3} R\left(t^{\prime \prime}, t\right)^{3}+\int_{t_{c}}^{t^{\prime}} d t^{\prime \prime} \Gamma\left(t^{\prime \prime}\right) V_{\cap}\right]$.

The intersection volume $V_{\cap}$ depends on the radii

$$
r \equiv R\left(t^{\prime \prime}, t\right), \quad r^{\prime} \equiv R\left(t^{\prime \prime}, t^{\prime}\right)
$$

and on the separation $s$ between $p$ and $p^{\prime}$. It is given by 


$$
V_{\cap}= \begin{cases}4 \pi r^{\prime 3} / 3 & \text { for } s \leq r-r^{\prime}, \\ \frac{\pi}{12}\left(r+r^{\prime}-s\right)^{2}\left[s+2\left(r+r^{\prime}\right)-\frac{3\left(r-r^{\prime}\right)^{2}}{s}\right] & \text { for } r-r^{\prime}<s \leq r+r^{\prime}, \\ 0 & \text { for } s>r+r^{\prime} .\end{cases}
$$

Notice that, if the separation is small enough, the smaller sphere is completely contained inside the larger one, ${ }^{3}$ hence the value $4 \pi r^{\prime 3} / 3$. On the other hand, if the separation is large enough, the intersection is empty and we have $V_{\cap}=0$ (see Fig. 2). Finally, we write Eq. (11) in the form

$$
P_{p \mid p^{\prime}}\left(t, t^{\prime}, s\right)=\exp \left[-I(t)+I_{\cap}\left(t, t^{\prime}, s\right)\right],
$$

where the function $I(t)$ is given by Eq. (7), and we have defined the quantity

$$
I_{\cap}\left(t, t^{\prime}, s\right)=\int_{t_{c}}^{t^{\prime}} d t^{\prime \prime} \Gamma\left(t^{\prime \prime}\right) V_{\cap}\left(r, r^{\prime}, s\right)
$$

\section{Probability that multiple points remain in the false vacuum}

Although we are mostly interested in points on bubble walls, we shall comment on the probability for several arbitrary points to remain in the false vacuum. We have obtained the probability $P_{p \mid p^{\prime}}\left(t, t^{\prime}, s\right)$ of the point $p$ being in the false vacuum at time $t$, under the condition that $p^{\prime}$ was in the false vacuum at time $t^{\prime} \leq t$. Multiplying by the probability $P_{f v}\left(t^{\prime}\right)$ that $p^{\prime}$ was in the false vacuum at time $t^{\prime}$, Eq. (6), we obtain the joint probability ${ }^{4}$ that $p$ is in the false vacuum at time $t$ and $p^{\prime}$ is in the false vacuum at time $t^{\prime}$,

$$
\begin{aligned}
P_{f v}^{(2)}\left(t, t^{\prime}, s\right) & =P_{f v}\left(t^{\prime}\right) P_{p \mid p^{\prime}}\left(t, t^{\prime}, s\right) \\
& =\exp \left[-I(t)-I\left(t^{\prime}\right)+I_{\cap}\left(t, t^{\prime}, s\right)\right]
\end{aligned}
$$

(we denote the two-point case with a superscript 2). The exponent in the last expression can be written as $-I_{\cup}$, with

$$
I_{\cup}\left(t, t^{\prime}, s\right)=\int_{t_{c}}^{t} d t^{\prime \prime} \Gamma\left(t^{\prime \prime}\right) V_{\cup}
$$

\footnotetext{
${ }^{3}$ Remember that we are considering the specific case $t^{\prime} \leq t$, so we have $r^{\prime} \leq r$. For $t^{\prime}>t$ (in which case the probability $P_{p \mid p^{\prime}}$ is conditioned to the point $p^{\prime}$ being in the false vacuum in the future) the calculation is similar, and the result is essentially the same. To take into account this possibility, the limit of integration $t^{\prime}$ in the second integral of Eq. (11) must be replaced with $t_{m}=\min \left\{t, t^{\prime}\right\}$.

${ }^{4}$ For this joint probability, there is no loss of generality in the assumption $t^{\prime} \leq t$.
}

where $V_{\cup}$ is the volume of the union of the two spheres of radii $R\left(t^{\prime \prime}, t\right)$ and $R\left(t^{\prime \prime}, t^{\prime}\right)$,

$$
V_{\cup}=\frac{4 \pi}{3} r^{3}+\left[\frac{4 \pi}{3} r^{\prime 3}+V_{\cap}\left(r, r^{\prime}, s\right)\right] \Theta\left(t^{\prime}-t^{\prime \prime}\right)
$$

This expression takes into account the fact that there is no sphere of radius $R\left(t^{\prime \prime}, t^{\prime}\right)$ for $t^{\prime \prime}>t^{\prime}$.

We could have obtained this result as a generalization of the calculation of $P_{f v}^{(1)}(t) \equiv P_{f v}(t)$. In this case, $P_{\text {out }}\left(t^{\prime \prime}\right)$ would denote the probability that none of the two points $p$, $p^{\prime}$ has been eaten by bubbles nucleated before time $t^{\prime \prime}$, and $d P\left(t^{\prime \prime}\right)$ the probability that at least one of them has been reached by a bubble nucleated between $t^{\prime \prime}$ and $t^{\prime \prime}+d t^{\prime \prime}$. This leads to the total volume $V_{\cup}$. ${ }^{5}$ The generalization to the probability that $n$ points $p_{1}, \ldots, p_{n}$ remain in the false vacuum at times $t_{1}, \ldots, t_{n}$, respectively, is straightforward. For a bubble nucleated at time $t^{\prime \prime}$ to reach any of the points $p_{i}$ before the corresponding time $t_{i}$, the bubble must have nucleated within one of the spheres of radius $R\left(t^{\prime \prime}, t_{i}\right)$ centered at $p_{i}$. This is illustrated in Fig. 3 for the case of three points. The result involves the volume $V_{\cup}$ of the union of the $n$ spheres, which depends on the separations $s_{i j}$ between the different points $p_{i}$ as well as on the radii $R\left(t^{\prime \prime}, t_{i}\right)$. Thus, we have

$$
P_{f v}^{(n)}\left(t_{i}, s_{i j}\right)=e^{-I_{\cup}\left(t_{i}, s_{i j}\right)},
$$

where $I_{\cup}=\int_{t_{c}}^{t_{\text {max }}} d t^{\prime \prime} \Gamma\left(t^{\prime \prime}\right) V_{\cup}$ and $t_{\max }=\max \left\{t_{i}\right\}$. The computation of $V_{\cup}$ must take into account that for $t^{\prime \prime}>t_{i}$ we have $R\left(t^{\prime \prime}, t_{i}\right)=0$. Care must also be taken of avoiding overcounting the intersections, which may be multiple.

\section{POINTS ON BUBBLE WALLS}

The calculations of the previous section can be adapted to points on bubble walls. Considering the bubbles as overlapping spheres, a given point of a wall has not collided

\footnotetext{
${ }^{5}$ See also Ref. [32] for an alternative calculation of $P_{f v}^{(2)}$ using past light cones of the two events $(t, p),\left(t^{\prime}, p^{\prime}\right)$. Although a constant velocity was assumed there, the derivation is valid in general, and our result (16)-(18) should be equivalent to Eqs. (42)-(45) of that paper. Indeed, this can be verified by comparing Eqs. (46)-(47) of [32] with our Eq. (74) for the specific case of an exponential nucleation rate, which we consider in Sec. IV.
} 


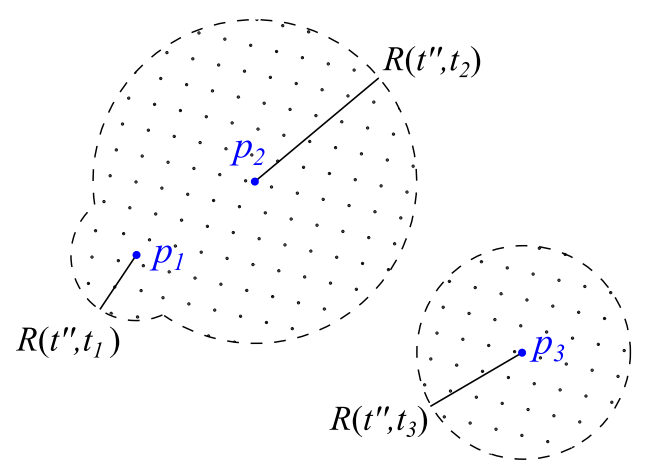

FIG. 3. The region in which bubbles must nucleate at time $t^{\prime \prime}$ in order to reach at least one of the points $p_{i}$ before time $t_{i}$.

if it has not been eaten by another bubble. For joint probabilities, perhaps the most direct approach is to consider, like in Sec. II D, the whole region of bubble nucleations at time $t^{\prime \prime}$ (the dotted region in Fig. 3). However, we are also interested in conditional probabilities, so we shall follow the steps of Sec. II C.

\section{A. Probability that a point of a bubble wall remains uncollided}

It is instructive to consider first the simpler case of a single point, which was first discussed in Ref. [1]. Since a single bubble has a negligible contribution to the fraction of volume occupied by bubbles, it seems, at first sight, that the probability of a given point $p$ on its surface remaining uncollided at time $t$ will be given by the fraction of volume $P_{f v}(t)$. However, the presence of the reference bubble to which $p$ is attached modifies the probability that $p$ remains in the false vacuum.

Like in Sec. II B, we begin by considering the probability that the point $p$ is outside of any bubble nucleated before some time $t^{\prime \prime}<t$. This leads to the differential Eq. (2) and its solution (4). Thus, we obtain the probability that $p$ is uncollided,

$$
P_{u}=e^{-\int_{t_{c}}^{t} d P\left(t^{\prime \prime}\right)},
$$

where, like before, $d P\left(t^{\prime \prime}\right)$ is the probability of $p$ being inside a bubble nucleated between $t^{\prime \prime}$ and $t^{\prime \prime}+d t^{\prime \prime}$, assuming that it is not inside any bubble nucleated before $t^{\prime \prime}$. Again, for this to happen, a bubble must have nucleated at a distance smaller than $R\left(t^{\prime \prime}, t\right)$ from $p$ (the dotted region in Fig. 1). For the present case, Fig. 4 shows the dotted region as well as the wall which contains $p$ (represented with a solid red line). The corresponding bubble was nucleated at a certain time $t_{N}$, and we may have $t_{N}<t^{\prime \prime}$ or $t_{N}>t^{\prime \prime}$.

We need to determine which part of the dotted region is actually available for bubble nucleation at time $t^{\prime \prime}$. It is straightforward to show that, like in the previous section, the dotted region could not be invaded at time $t^{\prime \prime}$ by bubbles nucleated at previous times ${ }^{6} t_{\text {prev }}<t^{\prime \prime}$. On the other hand, in the case $t^{\prime \prime}<t_{N}$, a bubble nucleated at $t^{\prime \prime}$ may prevent the nucleation of the reference bubble $B$. This will happen if the former nucleates too close to the nucleation point of the latter; specifically, within a radius $R\left(t^{\prime \prime}, t_{N}\right)$ (shaded region in the right panel of Fig. 4). Since we are assuming that bubble $B$ exists, no bubbles can have nucleated in this region at time $t^{\prime \prime}$. The probability that a bubble nucleates in the remaining part of the dotted region at a time between $t^{\prime \prime}$ and $t^{\prime \prime}+d t^{\prime \prime}$ is given $\mathrm{by}^{7}$

$$
d P\left(t^{\prime \prime}\right)=d t^{\prime \prime} \Gamma\left(t^{\prime \prime}\right) \frac{4 \pi}{3}\left[R\left(t^{\prime \prime}, t\right)^{3}-R\left(t^{\prime \prime}, t_{N}\right)^{3}\right],
$$

for $t^{\prime \prime}<t_{N}$. In contrast, for $t^{\prime \prime}>t_{N}$, the whole dotted region is available, and we have

$$
d P\left(t^{\prime \prime}\right)=d t^{\prime \prime} \Gamma\left(t^{\prime \prime}\right) \frac{4 \pi}{3} R\left(t^{\prime \prime}, t\right)^{3} .
$$

From (20)-(22) we obtain

$$
P_{u}\left(t, t_{N}\right)=\exp \left[-I(t)+I\left(t_{N}\right)\right] .
$$

The probability $P_{u}$ gives also the fraction of points on the wall of the bubble nucleated at $t_{N}$, which are still in the false vacuum at time $t$, i.e., the uncollided fraction of its surface. The result is $P_{u}=P_{f v}(t) / P_{f v}\left(t_{N}\right)$, which has a simple interpretation. Consider a large volume $V$. Inside this volume, a nucleation at time $t_{N}$ can only occur in the available volume $V P_{f v}\left(t_{N}\right)$. The nucleated bubble is initially uncollided. For very large $V$, the probability that part of this single bubble leaves the volume $V P_{f v}\left(t_{N}\right)$ at later times is negligible. Nevertheless, this initial volume is invaded due to the nucleation and growth of many other bubbles, and, by time $t$, a smaller part of it, $V P_{f v}(t)$, remains in the false vacuum. Thus, the reference bubble is still contained in the initial volume but, in average, only a fraction $V P_{f v}(t) / V P_{f v}\left(t_{N}\right)$ of its points remains in the false vacuum region. This alternative derivation gives also the fraction of the bubble volume that is not covered by other bubbles.

\section{B. Probability that two points of a bubble wall remain uncollided}

We now consider two points $p$ and $p^{\prime}$ on the surface of a bubble $B$ nucleated at time $t_{N}$. We shall first find the conditional probability that $p$ remains in the false vacuum

\footnotetext{
${ }^{6}$ In particular, in the case $t_{N}<t^{\prime \prime}$, the reference bubble wall containing $p$ will be, at time $t^{\prime \prime}$, just touching the limit of the dotted region, since $R\left(t_{N}, t^{\prime \prime}\right)+R\left(t^{\prime \prime}, t\right)=R\left(t_{N}, t\right)$. This is sketched with a red dotted circle in the left panel of Fig. 4.

${ }^{7}$ The forbidden (shaded) region is always completely contained inside the sphere with dots, since $R\left(t^{\prime \prime}, t_{N}\right)+R\left(t_{N}, t\right)=$ $R\left(t^{\prime \prime}, t\right)$.
} 

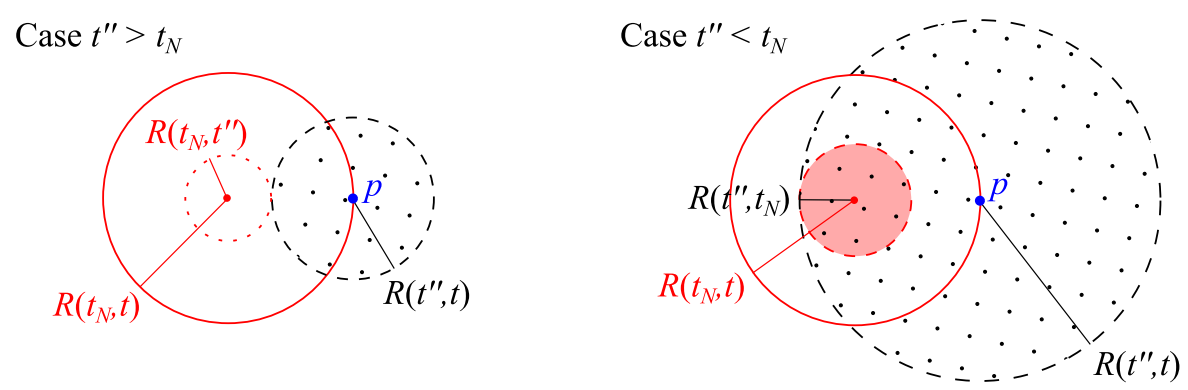

FIG. 4. A bubble $B$ nucleated at time $t_{N}$ (in red) whose wall contains the point $p$, and the region where bubbles must nucleate at time $t^{\prime \prime}$ (dots) in order to reach $p$ before time $t$. The shaded region corresponds to nucleations at $t^{\prime \prime}$ which would prevent the nucleation of the reference bubble.
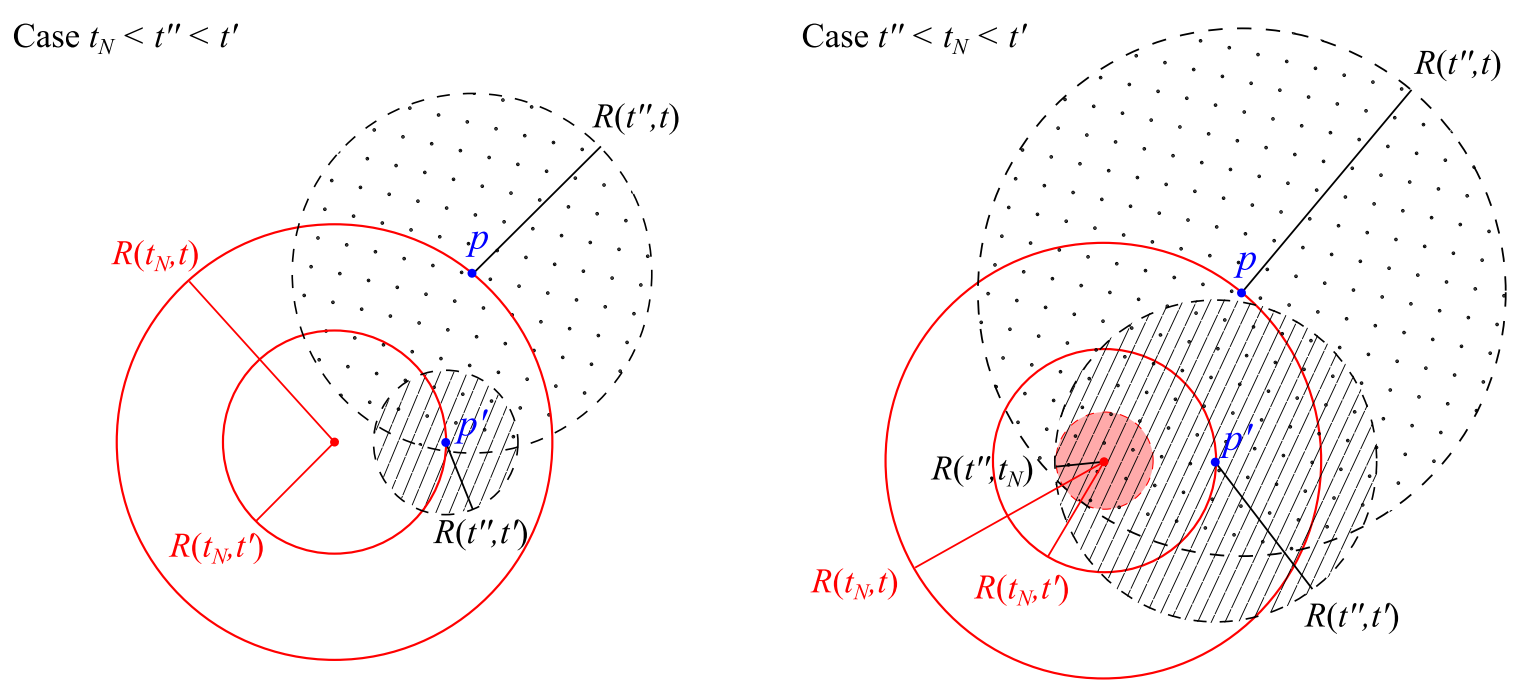

FIG. 5. The reference bubble $B$ nucleated at time $t_{N}$, at two subsequent times $t^{\prime}$ and $t$ (in red). The dots represent the nucleations at time $t^{\prime \prime}$, which affect the point $p$ before time $t$. Nucleations at $t^{\prime \prime}$ in the striped region would eat the point $p^{\prime}$ before time $t^{\prime}$, and those in the shaded region would prevent the nucleation of $B$.

at time $t$, given that $p^{\prime}$ was in the false vacuum at time $t^{\prime}$. Following the same steps of Sec. II C, we obtain again

$$
P_{p \mid p^{\prime}}^{S}=e^{-\int_{t_{c}}^{t} d P\left(t^{\prime \prime}\right)}
$$

(the superscript $S$ indicates that the two points belong to the surface of the bubble). We only need to recalculate the probability $d P\left(t^{\prime \prime}\right)$ of $p^{\prime}$ being outside of any bubble nucleated before $t^{\prime \prime}$ and inside a bubble nucleated between $t^{\prime \prime}$ and $t^{\prime \prime}+d t^{\prime \prime}$. Such a bubble must have nucleated within a sphere of radius $R\left(t^{\prime \prime}, t\right)$ centered at $p$ (the dotted region in previous figures). As we have already seen, under the above conditions the dotted region is not affected by bubbles nucleated at times $t_{\text {prev }}<t^{\prime \prime}$, but we must exclude those nucleation points that would prevent the nucleation of bubble $B$ at time $t_{N}$. Besides, since we are also assuming that the point $p^{\prime}$ is in the false vacuum at time $t^{\prime}$, we must also exclude nucleation points that would affect this event.
For the sake of concreteness, let us assume that $t^{\prime} \leq t$; the case $t^{\prime}>t$ is similar and gives essentially the same result. ${ }^{8}$ For $t^{\prime \prime}>t^{\prime}$, the nucleation at time $t^{\prime \prime}$ cannot affect events at times $t_{N}$ or $t^{\prime}$, so we have

$$
d P\left(t^{\prime \prime}\right)=d t^{\prime \prime} \Gamma\left(t^{\prime \prime}\right) \frac{4 \pi}{3} R\left(t^{\prime \prime}, t\right)^{3}
$$

The case $t^{\prime \prime}<t^{\prime}$ is sketched in Fig. 5. For $t^{\prime \prime}>t_{N}$ (left panel), a nucleation at time $t^{\prime \prime}$ cannot affect the nucleation of $B$ at time $t_{N}$ but may affect the point $p^{\prime}$ before time $t^{\prime}$. Hence, any nucleation at time $t^{\prime \prime}$ must occur at a distance larger than $R\left(t^{\prime \prime}, t^{\prime}\right)$ from $p^{\prime}$ (i.e., outside the striped region). For $t^{\prime \prime}<t_{N}$ (right panel), a nucleation at $t^{\prime \prime}$ may also affect the nucleation of the reference bubble. This will only happen if the nucleation at $t^{\prime \prime}$ occurs within a radius $R\left(t^{\prime \prime}, t_{N}\right)$ from the center of $B$ (shaded region).

\footnotetext{
${ }^{8}$ See footnote 3 .
} 


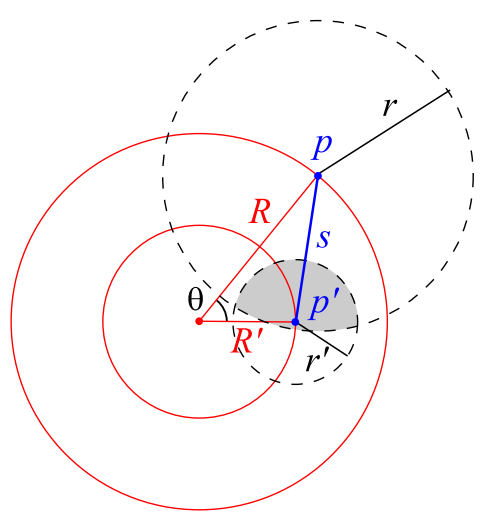

FIG. 6. The intersection volume $V_{\cap}$ and the separation $s$. The configuration corresponds to the example on the left of Fig. 5.

Nevertheless, this region is fully contained in the striped region. ${ }^{9}$ Therefore, we only have to exclude the striped region from the dotted one. Thus, for $t^{\prime \prime}<t^{\prime}$ we have

$$
d P\left(t^{\prime \prime}\right)=d t^{\prime \prime} \Gamma\left(t^{\prime \prime}\right)\left[\frac{4 \pi}{3} R\left(t^{\prime \prime}, t\right)^{3}-V_{\cap}\left(r, r^{\prime}, s\right)\right],
$$

where $V_{\cap}$ is the volume of the intersection of the dotted and striped regions, given by Eq. (13).

From Eqs. (24)-(26), we obtain the probability of the point $p$ being in the false vacuum at time $t$ under the condition that $p^{\prime}$ is in the false vacuum at time $t^{\prime}$,

$$
P_{p \mid p^{\prime}}^{S}\left(t, t^{\prime}, s\right)=\exp \left[-I(t)+I_{\cap}\left(t, t^{\prime}, s\right)\right],
$$

with $I_{\cap}$ given by Eq. (15). The result coincides with Eq. (14), which corresponds to the case of two arbitrary points in space. Here, the condition that $p$ is attached to a bubble does not have more implications than the condition that $p^{\prime}$ (on the same bubble) is uncollided. ${ }^{10}$ Multiplying Eq. (27) by the probability that $p^{\prime}$ was uncollided at time $t^{\prime}$, Eq. (23), we obtain the joint probability

$P_{p, p^{\prime}}^{S}\left(t, t^{\prime}, t_{N}, s\right)=\exp \left[-I(t)-I\left(t^{\prime}\right)+I\left(t_{N}\right)+I_{\cap}\left(t, t^{\prime}, s\right)\right]$.

As we have seen, the intersection volume $V_{\cap}$ depends on the distances $r=R\left(t^{\prime \prime}, t\right)$ and $r^{\prime}=R\left(t^{\prime \prime}, t^{\prime}\right)$, and on the separation $s$. The latter can be written as a function of the bubble radii

$$
R \equiv R\left(t_{N}, t\right), \quad R^{\prime} \equiv R\left(t_{N}, t^{\prime}\right),
$$

\footnotetext{
${ }^{9}$ Since $R\left(t^{\prime \prime}, t_{N}\right)+R\left(t_{N}, t^{\prime}\right)=R\left(t^{\prime \prime}, t^{\prime}\right)$. In other words, the shaded region also affects the point $p^{\prime}$ and is already taken into account.

${ }^{10}$ The result would be different if $p^{\prime}$ were a random point in space. Below we consider a similar case, namely, when $p^{\prime}$ belongs to a different bubble $B^{\prime}$.
}

and the angle $\theta$ between the positions of the points $p$ and $p^{\prime}$ relative to the bubble center (see Fig. 6),

$$
s=\sqrt{R^{2}+R^{\prime 2}-2 R R^{\prime} \cos \theta} .
$$

We thus have $R-R^{\prime} \leq s \leq R+R^{\prime}$. As we have seen in Sec. II C, for $s \leq r-r^{\prime}$ we have $V_{\cap}=4 \pi r^{\prime 3} / 3$. In the present case, in which the two points belong to the same bubble wall, we will never actually have $s<r-r^{\prime}$. Indeed, notice that

$$
r-r^{\prime}=R\left(t^{\prime}, t\right)=R-R^{\prime} \leq s .
$$

On the other hand, we may have $r+r^{\prime}>s$, for which $V_{\cap}=0$, so we write

$$
\begin{aligned}
V_{\cap}= & \frac{\pi}{12}\left(r+r^{\prime}-s\right)^{2}\left[s+2\left(r+r^{\prime}\right)-\frac{3\left(r-r^{\prime}\right)^{2}}{s}\right] \\
& \times \Theta\left(r+r^{\prime}-s\right) .
\end{aligned}
$$

\section{Points on walls of different bubbles}

Now we consider the case in which the two points $p$ and $p^{\prime}$ belong to the walls of two different bubbles $B$ and $B^{\prime}$, nucleated at times $t_{N}$ and $t_{N}^{\prime}$, respectively.

\section{General considerations}

There are some conditions which will have to be taken into account eventually. In the first place, we assume that both reference bubbles exist, so neither bubble should be occupying the nucleation center of the other one. This implies that the distance $l$ between the bubble centers must be larger than the distance travelled by a wall from one center to the other,

$$
l>\left|R\left(t_{N}, t_{N}^{\prime}\right)\right| .
$$

In the second place, if the bubbles are too close, it may happen, for instance, that the point $p^{\prime}$ by time $t^{\prime}$ is already inside the bubble $B$. This case will be forbidden from the beginning when we consider a conditional probability that assumes that $p^{\prime}$ is uncollided at that time. On the other hand, when we consider the joint probability for both points to be uncollided at the corresponding times, the situation is not forbidden, but its probability vanishes. We shall assume that we are not in this situation, which implies the condition

$$
d^{\prime}>R\left(t_{N}, t^{\prime}\right)
$$

where $d^{\prime}$ is the distance from the point $p^{\prime}$ to the center of the bubble $B$. Similarly, requiring that the point $p$ is not inside the bubble $B^{\prime}$ by time $t$, we have the condition 

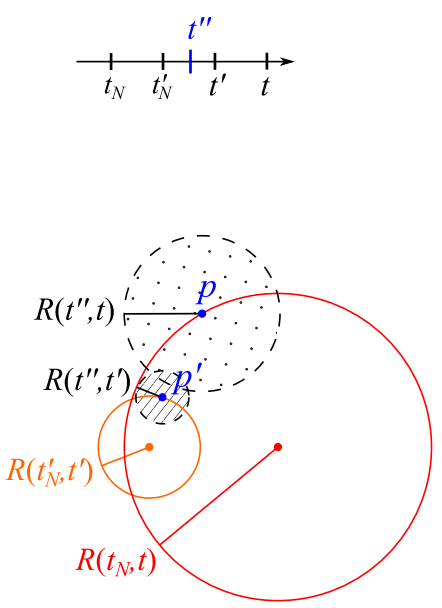
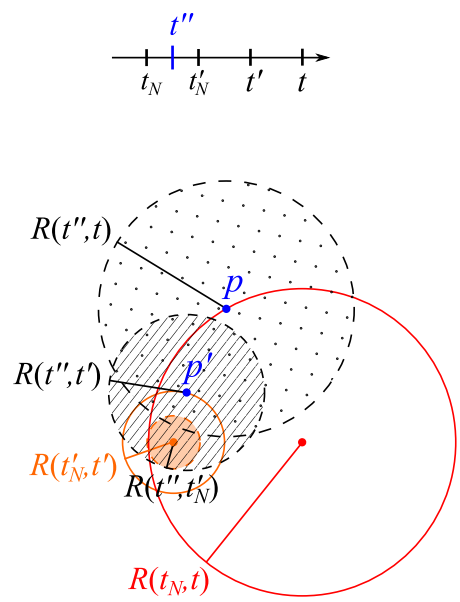
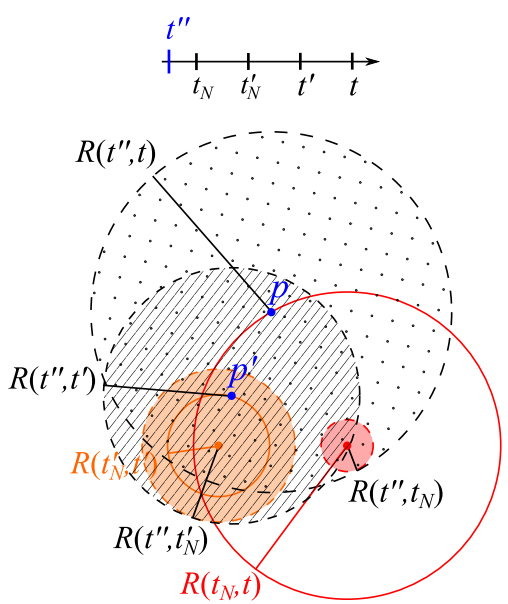

FIG. 7. A bubble $B$ nucleated at time $t_{N}$ (red) and a bubble $B^{\prime}$ nucleated at time $t_{N}^{\prime}$ (orange). The former is drawn at time $t$ and the latter, at time $t^{\prime}$, with the points $p$ and $p^{\prime}$ on each bubble surface. The black dots indicate the nucleations at time $t^{\prime \prime}$, which affect $p$ at time $t$. Those which fall inside the striped region would also affect $p^{\prime}$ at time $t^{\prime}$, and those in the shaded regions would affect the nucleations of $B$ or $B^{\prime}$.

$$
d>R\left(t_{N}^{\prime}, t\right)
$$

where $d$ is the distance from $p$ to the center of $B^{\prime}$. These two conditions together imply Eq. (33). ${ }^{11}$ These restrictions do not affect the discussions on the nucleations at time $t^{\prime \prime}$ below, and the examples shown in the figures fulfill them. Nevertheless, in applying our results, it should be taken into account that the probability vanishes beyond the limits imposed by these conditions.

\section{Probability that a point of a bubble wall is uncollided, given that a point of another bubble wall is uncollided}

First, we assume that $p^{\prime}$ is uncollided at time $t^{\prime}$, and we calculate the probability that $p$ is uncollided at time $t$. For the sake of concreteness we shall consider only the case ${ }^{12}$ $t \geq t^{\prime}$, but we must consider the two possibilities $t_{N}^{\prime}<t_{N}$ and $t_{N}<t_{N}^{\prime}$. Thus, there are three possible time orderings, namely, $t_{N}^{\prime}<t^{\prime}<t_{N}, t_{N}^{\prime}<t_{N}<t^{\prime}$, or $t_{N}<t_{N}^{\prime}<t^{\prime}$ (the latter is considered in Fig. 7). The conditional probability is again given by

$$
P_{p \mid p^{\prime}}^{S S^{\prime}}\left(t, t^{\prime}\right)=e^{-\int_{t_{c}}^{t} d P\left(t^{\prime \prime}\right)}
$$

(the superscript $S S^{\prime}$ indicates that the points belong to the surfaces $S$ and $S^{\prime}$ of two different bubbles), and we must compute the probability $d P\left(t^{\prime \prime}\right)$ that $p$ has not been reached by bubbles nucleated before time $t^{\prime \prime}$ but has been reached

\footnotetext{
${ }^{11}$ Let us denote $\mathbf{l}$ as the vector going from the center of $B$ to that of $B^{\prime}, \mathbf{R}$ as the vector joining the center of $B$ with $p$, and $\mathbf{R}^{\prime}$ as the vector joining the center of $B^{\prime}$ with $p^{\prime}$. We have $R=R\left(t_{N}, t\right)$, $R^{\prime}=R\left(t_{N}^{\prime}, t^{\prime}\right), d=|\mathbf{I}-\mathbf{R}|$, and $d^{\prime}=\left|\mathbf{l}+\mathbf{R}^{\prime}\right|$ (see Fig. 8). Then, the triangular inequality gives $d \leq l+R$ and $d^{\prime} \leq l+R^{\prime}$. Inserting these inequalities in Eqs. (35)-(34) gives Eq. (33).

${ }^{12}$ See footnote 3 .
}

by a bubble nucleated in the interval $\left[t^{\prime \prime}, t^{\prime \prime}+d t^{\prime \prime}\right]$. As before, the nucleation at $t^{\prime \prime}$ must occur within a sphere of radius $R\left(t^{\prime \prime}, t\right)$ centered at $p$ (dotted region). However, some of these nucleations will also affect the point $p^{\prime}$ or the nucleations of the bubbles $B$ or $B^{\prime}$ and must be excluded.

Let us consider the time sequence $t_{N}<t_{N}^{\prime}<t^{\prime}<t$. The other cases are similar and lead to the same conclusion (see the Appendix). Figure 7 shows examples of the bubble configuration, corresponding to particular positions of the time $t^{\prime \prime}$ relative to the other times (shown in the timelines on top of each figure). The case $t^{\prime \prime}>t^{\prime}$ is the simplest one and is not shown in Fig. 7. In this case, the nucleation at $t^{\prime \prime}$ cannot affect the events at times $t^{\prime}, t_{N}^{\prime}$ or $t_{N}$, and we have the whole dotted volume. Hence,

$$
d P\left(t^{\prime \prime}\right)=d t^{\prime \prime} \Gamma\left(t^{\prime \prime}\right) \frac{4 \pi}{3} R\left(t^{\prime \prime}, t\right)^{3}, \quad\left(t^{\prime \prime}>t^{\prime}\right) .
$$

In the case $t_{N}^{\prime}<t^{\prime \prime}<t^{\prime}$ (left panel of Fig. 7), a bubble nucleated at time $t^{\prime \prime}$ may have eaten the point $p^{\prime}$ by time $t^{\prime}$, so we must exclude the sphere of radius $R\left(t^{\prime \prime}, t^{\prime}\right)$ centered at $p^{\prime}$ (striped region). We thus have

$d P\left(t^{\prime \prime}\right)=d t^{\prime \prime} \Gamma\left(t^{\prime \prime}\right)\left[\frac{4 \pi}{3} R\left(t^{\prime \prime}, t\right)^{3}-V_{\cap}\right], \quad\left(t_{N}^{\prime}<t^{\prime \prime}<t^{\prime}\right)$,

where $V_{\cap}$ is the volume of the intersection of the striped and dotted spheres, which is given by Eq. (13).

For $t_{N}<t^{\prime \prime}<t_{N}^{\prime}$ (shown in the central panel of Fig. 7), the nucleation at $t^{\prime \prime}$ may also prevent the nucleation of bubble $B^{\prime}$. Nevertheless, like in the previous section, the region that can affect this event (light orange shade in Fig. 7) is completely contained within the striped region, which is already excluded in Eq. (38). Therefore, nothing changes when $t^{\prime \prime}$ becomes smaller than $t_{N}^{\prime}$, 


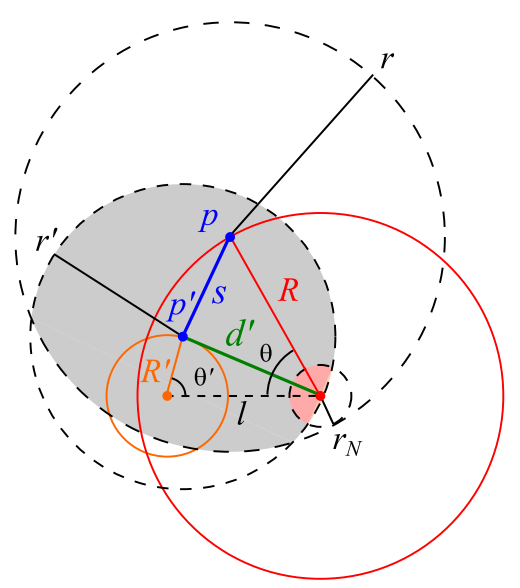

FIG. 8. The intersection volumes $V_{\cap}$ (gray) and $V_{\cap}^{\prime}$ (pink), and the distances $s$ and $d^{\prime}$. This specific configuration corresponds to the case on the right panel of Fig. 7.

$d P\left(t^{\prime \prime}\right)=d t^{\prime \prime} \Gamma\left(t^{\prime \prime}\right)\left[\frac{4 \pi}{3} R\left(t^{\prime \prime}, t\right)^{3}-V_{\cap}\right],\left(t_{N}<t^{\prime \prime}<t_{N}^{\prime}\right)$.

Finally, for $t^{\prime \prime}<t_{N}$ (right panel), the nucleation at $t^{\prime \prime}$ may also prevent the nucleation of bubble $B$. Therefore, we must exclude the sphere of radius $R\left(t^{\prime \prime}, t_{N}\right)$ around the center of $B$ (pink shade), as well as the striped region. We thus have

$$
\begin{aligned}
d P\left(t^{\prime \prime}\right)= & d t^{\prime \prime} \Gamma\left(t^{\prime \prime}\right)\left[\frac{4 \pi}{3} R\left(t^{\prime \prime}, t\right)^{3}-\frac{4 \pi}{3} R\left(t^{\prime \prime}, t_{N}\right)^{3}\right. \\
& \left.-V_{\cap}+V_{\cap}^{\prime}\right], \quad\left(t^{\prime \prime}<t_{N}\right) .
\end{aligned}
$$

Here, we have first subtracted the volume of the pink region, which is completely contained inside the dotted region, then we have subtracted the intersection volume $V_{n}$ of the striped and dotted regions. The volume $V_{n}^{\prime}$ is a correction for the case in which the striped region overlaps with the pink region, like in the example of Fig. 7. This volume must be added in order to avoid subtracting twice their intersection. This happens when the distance $d^{\prime}$ between $p^{\prime}$ and the center of $B$ is short enough. Thus, $V_{\cap}^{\prime}$ depends on $d^{\prime}$ and on the radii of the two spheres, $r^{\prime}=$ $R\left(t^{\prime \prime}, t^{\prime}\right)$ and $r_{N} \equiv R\left(t^{\prime \prime}, t_{N}\right)$ (see Fig. 8), and we have ${ }^{13}$

$$
\begin{aligned}
V_{\cap}^{\prime}= & \frac{\pi}{12}\left(r^{\prime}+r_{N}-d^{\prime}\right)^{2}\left[d^{\prime}+2\left(r^{\prime}+r_{N}\right)-\frac{3\left(r^{\prime}-r_{N}\right)^{2}}{d^{\prime}}\right] \\
& \times \Theta\left(r^{\prime}+r_{N}-d^{\prime}\right) .
\end{aligned}
$$

\footnotetext{
${ }^{13}$ The Heaviside function takes into account the fact that for large enough separation the intersection is empty. On the other hand, the condition (34), $d^{\prime}>R\left(t_{N}, t^{\prime}\right)=R\left(t^{\prime \prime}, t^{\prime}\right)-R\left(t^{\prime \prime}, t_{N}\right)=$ $r^{\prime}-r_{N}$, implies that the sphere of radius $r_{N}$ will never be contained completely inside that of radius $r^{\prime}$, except as a limit.
}

Inserting these results in Eq. (36), we obtain

$$
\begin{aligned}
& P_{p \mid p^{\prime}}^{S S^{\prime}}\left(t, t^{\prime}, s, d^{\prime}, t_{N}\right) \\
& \quad=\exp \left[-I(t)+I\left(t_{N}\right)+I_{\cap}\left(t, t^{\prime}, s\right)-I_{\cap}^{\prime}\left(t^{\prime}, t_{N}, d^{\prime}\right)\right],
\end{aligned}
$$

where $I_{\cap}$ is the integral given by Eq. (15), and $I_{\cap}^{\prime}$ is a similar integral involving $V_{n}^{\prime}$. According to Eq. (40), the upper limit of this integral is $t_{N}$. However, if we take into account the possibility $t^{\prime}<t_{N}$ (not considered in the example used for this derivation), we must write (see the Appendix for details)

$$
I_{\cap}^{\prime}\left(t^{\prime}, t_{N}, d^{\prime}\right)=\int_{t_{c}}^{\min \left\{t_{N}, t^{\prime}\right\}} d t^{\prime \prime} \Gamma\left(t^{\prime \prime}\right) V_{\cap}^{\prime}\left(r^{\prime}, r_{N}, d^{\prime}\right) .
$$

The distance $s$ between $p$ and $p^{\prime}$ is no longer given by Eq. (30). We may relate the relevant distances with the orientations of the points on each bubble surface,

$$
\begin{gathered}
s^{2}=l^{2}+R^{2}+R^{\prime 2}-2 l R^{\prime} \cos \theta^{\prime}-2 l R \cos \theta \\
+2 R R^{\prime}\left(\sin \theta \sin \theta^{\prime} \cos \phi-\cos \theta \cos \theta^{\prime}\right), \\
d^{\prime}=\sqrt{R^{\prime 2}+l^{2}-2 R^{\prime} l \cos \theta^{\prime}}, \\
d=\sqrt{R^{2}+l^{2}-2 R l \cos \theta},
\end{gathered}
$$

where $R \equiv R\left(t_{N}, t\right), R^{\prime} \equiv R\left(t_{N}^{\prime}, t^{\prime}\right), \quad l$ is the separation between the bubble centers, the angles $\theta$ and $\theta^{\prime}$ (which are in the interval $[0, \pi]$ ) correspond to the orientations of the points $p$ and $p^{\prime}$ on each bubble with respect to the axis joining the two centers (see Fig. 8), and $\phi$ (in the interval $[0,2 \pi])$ is the angle between the projections of these directions on the plane perpendicular to the axis. Although Eq. (42) does not depend on $d$, this distance appears in the condition (35). Indeed, in the derivation of $P_{p \mid p^{\prime}}^{S S^{\prime}}$ we have assumed that the conditions (34)-(35) are fulfilled. The assumption $d^{\prime}>R\left(t_{N}, t^{\prime}\right)$ is correct, since the conditional probability assumes that the point $p^{\prime}$ is uncollided. On the other hand, the condition $d>R\left(t_{N}^{\prime}, t\right)$ is not necessarily valid. If it is not fulfilled, the point $p$ at time $t$ is inside bubble $B^{\prime}$, and the probability just vanishes, so we must multiply Eq. (42) by the Heaviside function

$$
\Theta\left(d-R\left(t_{N}^{\prime}, t\right)\right) .
$$

It is interesting to consider the limit in which $B$ and $B^{\prime}$ nucleate at the same time and very close to each other. For $l=0$, Eq. (45) gives $d^{\prime}=R^{\prime}$ and $s$ becomes the same as for the single-bubble case, i.e., Eq. (44) becomes Eq. (30). Besides, for $t_{N}=t_{N}^{\prime}$ we have $r^{\prime}-d^{\prime}=R\left(t^{\prime \prime}, t^{\prime}\right)-R\left(t_{N}^{\prime}, t^{\prime}\right)=$ $R\left(t^{\prime \prime}, t_{N}^{\prime}\right)=r_{N}^{\prime}=r_{N}$. Using this result, the volume $V_{n}^{\prime}$ becomes $V_{\cap}^{\prime}=\frac{4 \pi}{3} r_{N}^{3}$. Hence, $I_{\cap}^{\prime}$ cancels with $I\left(t_{N}\right)$ in Eq. (42), and we obtain $P_{p \mid p^{\prime}}^{S S^{\prime}}\left(t, t^{\prime}, s\right)=\exp [-I(t)+$ $\left.I_{\cap}\left(t, t^{\prime}, s\right)\right]$, which coincides with Eq. (28), i.e., the 
probability $P_{p \mid p^{\prime}}^{S}\left(t, t^{\prime}, s\right)$ for two points on the same bubble wall. This was to be expected, since in this limit the two bubbles are almost coincident. However, we must also take into account Eq. (46). In particular, in this limit many points on each surface must be eaten by the other bubble (here, we are assuming that $p^{\prime}$ is not). In the case $t_{N}^{\prime}=t_{N}$ Eq. (46) becomes $\Theta(d-R)$. For $l \rightarrow 0$ we have $d \rightarrow R$, so we must be careful with the limit. For $l \ll R$ Eqs. (45) can be written

$$
d-R=-l \cos \theta, \quad d^{\prime}-R^{\prime}=-l \cos \theta^{\prime} .
$$

Hence, the Heaviside function vanishes for $\cos \theta>0$, i.e., for $\theta<\pi / 2$. This is because, in this limit, a half of bubble $B$ is inside $B^{\prime}$.

\section{Probability that a point on a bubble wall is uncollided in the presence of another bubble}

To obtain the joint probability that a point $p$ on the surface of $B$ and a point $p^{\prime}$ on the surface of $B^{\prime}$ remain uncollided at times $t$ and $t^{\prime}$, respectively, we only have to multiply $P_{p \mid p^{\prime}}^{S S^{\prime}}$ by the probability that the point $p^{\prime}$ on the wall of $B^{\prime}$ is uncollided at time $t^{\prime}$ (without any condition on the point $p$ ). This probability was obtained in Sec. III A and is given by Eq. (23). However, the conditions are different in the present case, since we assume the existence of another bubble, $B$, at a certain distance from $B^{\prime}$ (otherwise, we cannot ask whether the point $p$ on $B$ is uncollided). Therefore, we must consider the probability that $p^{\prime}$ on $B^{\prime}$ is uncollided at time $t^{\prime}$, in the presence of the bubble $B$. This probability may be also of interest on its own.

Following the derivation of Sec. III A, we consider the region of nucleations at time $t^{\prime \prime}$ which affect $p^{\prime}$ at time $t^{\prime}$ (in Fig. 4 this was the dotted region, but in Fig. 7 it is represented by a striped region). Like before, we need to exclude nucleations at $t^{\prime \prime}$ which affect the nucleation of the reference bubble $B^{\prime}$ at $t_{N}^{\prime}$ (the orange region in Fig. 7), but also those which prevent the nucleation of $B$ at $t_{N}$ (the pink region). We thus obtain

$P_{p^{\prime} \mid B}^{u}\left(t^{\prime}, t_{N}^{\prime}, t_{N}, d^{\prime}\right)=\exp \left[-I\left(t^{\prime}\right)+I\left(t_{N}^{\prime}\right)+I_{\cap}^{\prime}\left(t^{\prime}, t_{N}, d^{\prime}\right)\right]$.

The first two terms in the exponent are like in Eq. (23). However, the probability that $p^{\prime}$ (on the surface of $B^{\prime}$ ) is uncollided depends also on its distance to the center of $B$ and the nucleation time of the latter. In this derivation we have assumed that Eq. (34) is fulfilled. Therefore, Eq. (48) does not take into account the possibility that bubble $B$ has eaten the point $p^{\prime}$, and we must add the factor

$$
\Theta\left(d^{\prime}-R\left(t_{N}, t^{\prime}\right)\right)
$$

\section{Probability that two points on the walls of different bubbles are uncollided}

The joint probability that both points are uncollided is given by the product of Eqs. (48) and (42),

$$
\begin{aligned}
& P_{p, p^{\prime}}^{S S^{\prime}}\left(t, t^{\prime}, t_{N}, t_{N}^{\prime}, s\right) \\
& \quad=\exp \left[-I(t)-I\left(t^{\prime}\right)+I\left(t_{N}\right)+I\left(t_{N}^{\prime}\right)+I_{\cap}\left(t, t^{\prime}, s\right)\right] .
\end{aligned}
$$

The integral $I_{\cap}^{\prime}\left(t^{\prime}, t_{N}, d^{\prime}\right)$ has canceled out, so this expression depends only on the point separation and not on the bubble separation $l$. The result is very similar to the singlebubble probability, Eq. (28), except for the extra term $I\left(t_{N}^{\prime}\right)$ in the exponent. However, we remark that if any of the conditions (34)-(35) is not fulfilled, one of the points has been eaten by the other bubble, and the probability actually vanishes. Therefore, Eq. (50) must be multiplied by the Heaviside functions

$$
\Theta\left(d-R\left(t_{N}^{\prime}, t\right)\right) \Theta\left(d^{\prime}-R\left(t_{N}, t^{\prime}\right)\right) .
$$

In particular, these conditions imply that the point separation is greater than the distance traveled by a bubble wall between the times $t^{\prime}$ and $t$, i.e., $s>R\left(t^{\prime}, t\right)$ (for $t^{\prime}<t$ ), which is essentially the same as Eq. (31), i.e., $r-r^{\prime}<s .{ }^{14}$ Therefore, the volume $V_{n}$, which is generally given by Eq. (13), can be written in the form (32).

To see the dependence with $l$, let us consider, for simplicity, the case $t_{N}^{\prime}=t_{N}$ and $t^{\prime}=t$, so that we have two bubbles of the same size. In this case, Eq. (51) becomes $\Theta(d-R) \Theta\left(d^{\prime}-R\right)$. For $l>R$, Eqs. (45) give $l-R<d<l+R$, and the same for $d^{\prime}$. For $l>2 R$, both $d$ and $d^{\prime}$ are larger than $R$, so the Heaviside functions give a factor of 1 . Thus, for large $l$, the probability is given by Eq. (50), which depends only on the point separation $s$. On the other hand, for $l<2 R$ the two bubbles overlap, and some points will have zero probability of being uncollided (depending on $d$ and $d^{\prime}$ ). For $l<R$, Eqs. (45) give $R-l<d<R+l$, and for $l \ll R$ we have Eqs. (47) which, inserted in (51) imply $\theta>\pi / 2, \theta^{\prime}>\pi / 2$. This is because, as already discussed, when the two bubbles almost coincide, a half of each bubble is inside the other bubble.

\section{UNCOLLIDED WALLS}

From the probabilities derived above we may obtain correlations between walls of different bubbles or different parts of a bubble wall. For concrete computations, we shall use a couple of simple models for $\Gamma(t)$ and $v(t)$, which we motivate below.

\footnotetext{
${ }^{14}$ The condition (35) implies $d>R\left(t_{N}^{\prime}, t^{\prime}\right)+R\left(t^{\prime}, t\right)=$ $R^{\prime}+R\left(t^{\prime}, t\right)$. On the other hand, the triangular inequality gives $d<R^{\prime}+s$. Comparing these two inequalities, we obtain $R\left(t^{\prime}, t\right)<$ $s$. It is also worth noting that in this case we have $R\left(t^{\prime}, t\right)=$ $r-r^{\prime} \neq R-R^{\prime}$.
} 


\section{A. Specific models}

In the first place, we shall assume that, after a bubble nucleates, its wall immediately reaches a terminal velocity. ${ }^{15}$ Assuming a homogeneous temperature, the time dependence of $\Gamma(T)$ and of $v(T)$ is determined by the function $T(t)$. The simplest possible model consists of a constant $\Gamma$ and a constant $v$. This model has been sometimes used as a simplification for computationally demanding approaches, such as those involving lattice calculations or other types of simulations (see, e.g., [30]). However, this model is hardly realistic, since the nucleation rate is very sensitive to the temperature, and the latter always varies during the phase transition.

For detonations or runaway walls, the reheating can be ignored until the end of the phase transition, so $T(t)$ decreases according to the adiabatic expansion. In this case, an exponentially growing nucleation rate is generally a good approximation. Although the wall velocity increases do to the temperature decrease, it can be assumed to be constant during the short time of bubble growth. This gives the widely used model

$$
\Gamma(t)=\Gamma_{*} e^{\beta t}, \quad R\left(t, t^{\prime}\right)=v\left(t^{\prime}-t\right) .
$$

For deflagrations, the evolution of $T$ is more involved (even assuming a homogeneous reheating). In general, there is a supercooling stage followed by a sudden reheating, after which a phase-equilibrium stage may occur. Due to the high sensitivity of the nucleation rate, $\Gamma$ turns off as soon as the reheating begins. In this case, a reasonable approximation is to assume that all bubbles nucleate at the moment $t_{*}$ at which $T$ reaches its minimum and $\Gamma$ reaches its maximum [40]. The wall velocity also decreases due to the reheating, but its evolution is less simple. Nevertheless, depending on the parameters, the velocity variation may not be significant and a constant velocity is a reasonable approximation (we shall consider other possibilities in the next section). In such a case, we have

$$
\Gamma(t)=n_{b} \delta\left(t-t_{*}\right), \quad R\left(t, t^{\prime}\right)=v\left(t^{\prime}-t\right) .
$$

This simple model has also been considered sometimes in time-demanding numerical computations (see, e.g., [41]).

The two models described by Eqs. (52) and (53) give phase transitions of a very different nature. For an exponentially growing nucleation rate, the growth of stablephase domains is dominated by bubble nucleation; while, in the case of simultaneous nucleation, the growth of the

\footnotetext{
${ }^{15}$ The time required to reach the terminal velocity is, in general, several orders of magnitude shorter than the total time of bubble expansion (see footnote 1) and can be neglected. This holds also in the runaway case, where the terminal velocity is $v=1$. Even in the case of wall corrugation mentioned below, this is a good approximation if the wall deformations are treated as small perturbations.
}

stable phase is dominated by bubble expansion. The kinematics of the former is thus characterized by the timescale $\beta^{-1}$, whereas for the latter the bubble density gives the characteristic length scale. It is therefore of interest to use these two cases as opposite examples. We shall now consider some basic quantities which are related to the physical consequences of the phase transition, and in the next section we shall discuss some specific applications.

\section{B. The envelope of uncollided walls}

For a given bubble of radius $R$, the locus of its uncollided wall is a subset of the sphere of radius $R$. A given point on the sphere is characterized by two angles $\theta, \phi$ by means of the parametrization $\mathbf{r}=R \hat{r}$, where $\hat{r}=(\sin \theta \cos \phi$, $\sin \theta \sin \phi, \cos \theta)$. The uncollided wall can be characterized by the indicator or characteristic function

$$
1_{S}(\theta, \phi)= \begin{cases}1 & \text { if } \mathbf{r} \in S, \\ 0 & \text { if } \mathbf{r} \notin S .\end{cases}
$$

Thus, the area of this bubble wall can be written in the form $^{16}$

$$
S=R^{2} \int_{0}^{2 \pi} d \phi \int_{0}^{\pi} d \theta \sin \theta 1_{S}(\theta, \phi) .
$$

If we regard the characteristic function as a stochastic variable and average over bubbles of the same radius $R$, we have

$$
\langle S\rangle=R^{2} \int_{0}^{2 \pi} d \phi \int_{0}^{\pi} \sin \theta d \theta\left\langle 1_{S}(\theta, \phi)\right\rangle .
$$

For each direction $\hat{r}$, we have two possible values of $1_{S}(\theta, \phi)$, with probabilities $P_{\hat{r}}(1)$ and $P_{\hat{r}}(0)=1-P_{\hat{r}}(1)$, and we have $\left\langle 1_{S}(\theta, \phi)\right\rangle=P_{\hat{r}}(1)$. This is the probability that the point represented by $\hat{r}$ is uncollided, which is given by Eq. (23) and is independent of the direction, $P_{\hat{r}}(1)=P_{u}\left(t, t_{N}\right)=e^{-I(t)+I\left(t_{N}\right)}$. Thus, the average uncollided area of a bubble of radius $R$ is given by

$$
\langle S\rangle=4 \pi R^{2} e^{-I(t)+I\left(t_{N}\right)} .
$$

To obtain the total surface in a given volume $V$, we must multiply Eq. (57) by the number of bubbles of radius $R$ in this volume, and then integrate over $R$. According to Eq. (1), the bubbles of radius $R$ are those that were nucleated at the time $t_{N}(R, t)$ such that $R=\int_{t_{N}}^{t} v\left(t^{\prime \prime}\right) d t^{\prime \prime}$. Thus, at time $t$, the bubbles that have radii between $R$ and $R+d R$ are those nucleated between $t_{N}-d t_{N}$ and $t_{N}$. The number of these bubbles is

\footnotetext{
${ }^{16}$ We use the same notation $S$ for the locus of the uncollided wall and its area.
} 

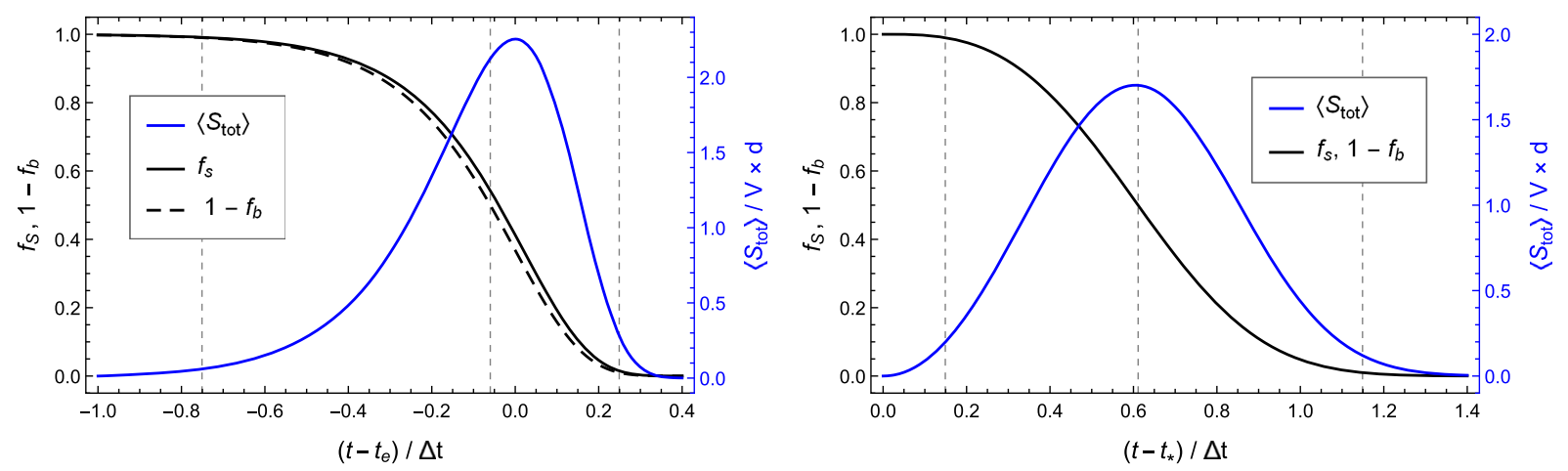

FIG. 9. The fraction of volume $P_{f v}=1-f_{b}$, the fraction of surface $f_{S}$, and the remaining wall area $\left\langle S_{\text {tot }}\right\rangle$ at time $t$ for the exponential nucleation (left) and for the simultaneous nucleation (right). The vertical lines correspond to the values $f_{b}=0.01,0.5$, and 0.99 .

$$
d N=\Gamma\left(t_{N}\right) V P_{f v}\left(t_{N}\right) d t_{N}
$$

Since $P_{f v}\left(t_{N}\right)=e^{-I\left(t_{N}\right)}$, we have

$$
\left\langle S_{\text {tot }}\right\rangle=\int d N\langle S\rangle=V e^{-I(t)} \int_{t_{c}}^{t} d t_{N} \Gamma\left(t_{N}\right) 4 \pi R\left(t_{N}, t\right)^{2}
$$

As discussed in Sec. I, for phenomena that depend on the bubble walls, the important measure of progress (rather than the volume fraction $f_{b}$ ) will be the fraction of uncollided wall area, $f_{S}(t)$, which is obtained by dividing Eq. (59) by $V \int d N 4 \pi R^{2}$,

$$
f_{S}(t)=\frac{e^{-I(t)} \int_{t_{c}}^{t} d t_{N} \Gamma\left(t_{N}\right) R\left(t_{N}, t\right)^{2}}{\int_{t_{c}}^{t} d t_{N} e^{-I\left(t_{N}\right)} \Gamma\left(t_{N}\right) R\left(t_{N}, t\right)^{2}} .
$$

In Ref. [1], the energy-weighted fraction $f_{E}(t)$ is also defined, by replacing $R\left(t_{N}, t\right)^{2}$ with $R\left(t_{N}, t\right)^{3}$ in the numerator and denominator of Eq. (60). It is to be expected that different measures of progress such as $f_{S}, f_{E}$, or $P_{f v}=$ $1-f_{b}$ (all of which vary from 1 to 0 throughout the phase transition) are qualitatively similar. In Ref. [1] it was found that, for the case of an exponentially growing nucleation rate and a constant velocity, $P_{f v}(t)$ and $f_{E}(t)$ are very similar even quantitatively. Let us consider the fraction of uncollided wall for this case. It is straightforward to calculate the integral (7), which gives $I(t)=8 \pi v^{3} \beta^{-4} \Gamma(t)$. We define the time $t_{e}$ at which $P_{f v}$ has decreased to $1 / e$, which is given by the condition $I\left(t_{e}\right)=1$. Thus, we have $I(t)=e^{\beta\left(t-t_{e}\right)}$, and we obtain

$$
\frac{\left\langle S_{\mathrm{tot}}\right\rangle}{V}=\frac{\beta}{v} e^{\beta\left(t-t_{e}\right)} \exp \left[-e^{\beta\left(t-t_{e}\right)}\right] .
$$

This quantity is proportional to the average nucleation rate $\bar{\Gamma}(t)=\Gamma(t) P_{f v}(t)$. It is easy to see that the maximum of $\left\langle S_{\text {tot }}\right\rangle$ (equivalently, the maximum of $\bar{\Gamma}$ ) occurs at $t=t_{e}$. The parameter $\beta^{-1}$ characterizes the timescale for this transition, and, if we measure the length in units of the associated scale $v \beta^{-1}$, the quantity $\left\langle S_{\text {tot }}\right\rangle / V$ does not depend on the wall velocity. In order to facilitate the comparison with the simultaneous nucleation model considered below, we shall also use sometimes a unit which corresponds to a physical time in the evolution of the phase transition. Specifically, we are going to consider the interval $\Delta t$ between the moments at which $f_{b}=0.01$ and $f_{b}=0.99$. We have $\Delta t=c \beta^{-1}$, with $c=\log (\log 0.01 / \log 0.99) \simeq$ 6.13. Accordingly, we shall measure the area and the volume in units of the associated distance $d=v \Delta t$. In the left panel of Fig. 9 we plot $\left\langle S_{\text {tot }}\right\rangle, f_{S}$ and $P_{f v}$ as functions of time.

For the delta-function rate (53) we have $I(t)=$ $n_{b} \frac{4 \pi}{3} R\left(t_{*}, t\right)^{3}$ and

$$
\left\langle S_{\text {tot }}\right\rangle=V n_{b} 4 \pi R\left(t_{*}, t\right)^{2} e^{-I(t)},
$$

while the denominator in Eq. (60) is given by $V n_{b} 4 \pi R\left(t_{*}, t\right)^{2}$. Therefore, we have $f_{S}(t)=e^{-I(t)}=$ $P_{f v}(t)$. The same happens with $f_{E}(t)$; i.e., for simultaneous nucleation all these measures of progress coincide. For this model, the natural unit of length is the characteristic distance $d_{b} \equiv n_{b}^{-1 / 3}$ (the "average" bubble separation), and, for constant velocity, the natural unit of time is the associated value $t_{b}=d_{b} / v$ (which gives an estimate for the duration of the phase transition). Thus, we may write

$$
f_{S}(t)=\exp \left[-\frac{4 \pi}{3}\left(\frac{t-t_{*}}{t_{b}}\right)^{3}\right]
$$

In these units, this function does not depend explicitly on the wall velocity. The average wall area is given by

$$
\frac{\left\langle S_{\mathrm{tot}}\right\rangle}{V}=\frac{4 \pi}{d_{b}}\left(\frac{t-t_{*}}{t_{b}}\right)^{2} \exp \left[-\frac{4 \pi}{3}\left(\frac{t-t_{*}}{t_{b}}\right)^{3}\right] .
$$

To compare with the exponential nucleation model, we shall also use the units $\Delta t$ and $d=v \Delta t$ corresponding to the time interval between the values $f_{b}=0.01$ and $f_{b}=0.99$. Inverting the relation $I(t)=\frac{4 \pi}{3}\left(R / d_{b}\right)^{3}$, we 

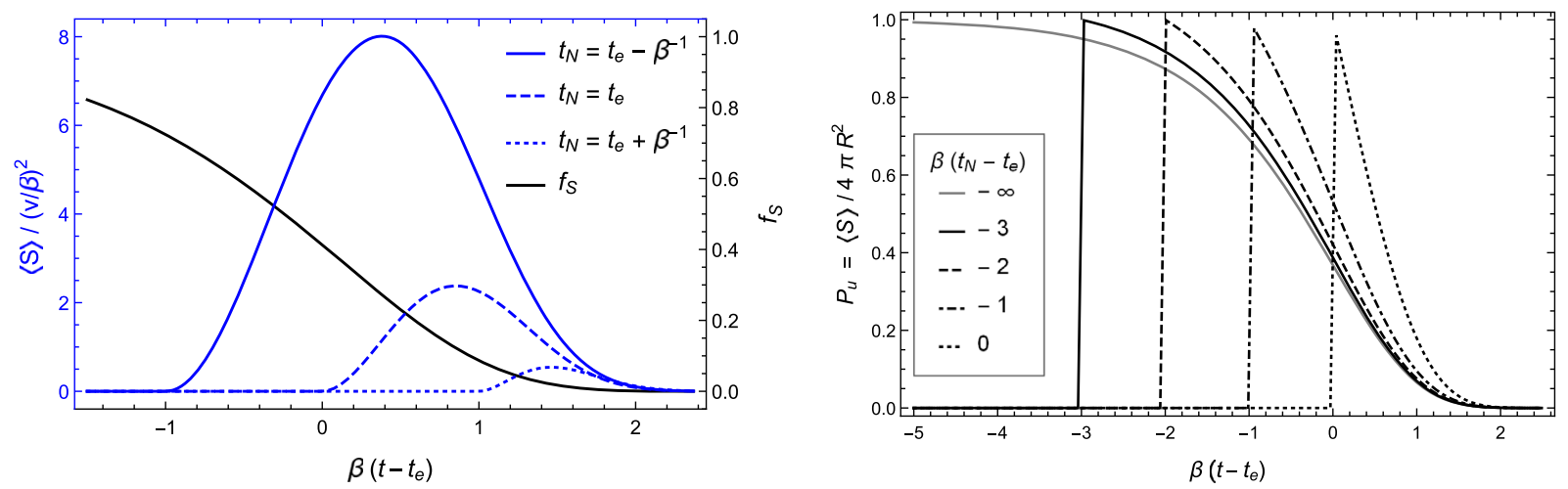

FIG. 10. Uncollided wall area of a bubble nucleated at time $t_{N}$ (left) and fraction of uncollided wall (right) for the exponential case.

have $t-t_{*}=t_{b}\left[-\frac{3}{4 \pi} \log \left(1-f_{b}\right)\right]^{1 / 3}$, and we obtain $\Delta t=\tilde{c} t_{b}$, with $\tilde{c} \simeq 0.9$. We plot the functions $\left\langle S_{\text {tot }}(t)\right\rangle$ and $f_{S}(t)$ in the right panel of Fig. 9.

We see that the width and the height of the curve of $\left\langle S_{\text {tot }}\right\rangle$ is, in these units, quantitatively similar for the two models. The simultaneous case gives a somewhat lower and wider curve, but such a precise comparison is not meaningful since these relations change if we use other units. For instance, if we use the average bubble separation $d_{b}$ and the corresponding timescale $d_{b} / v$ for the simultaneous case, we may define, for the exponential case, the bubble separation from the final bubble density, which gives $d_{b}=(8 \pi)^{1 / 3} \beta^{-1}$. It turns out that in these units the curve becomes lower and wider for the exponential case. This happens because the physical quantities $\Delta t$ or $d_{b}$, which are appropriate for the model comparison, are defined from the dynamics of each phase transition and are not absolute units of time or length.

Some other common features are the following. When bubbles occupy $1 \%$ of space, the area in their walls is already relatively high. This is more visible in the simultaneous case, where $\left\langle S_{\text {tot }}\right\rangle$ is more than a $10 \%$ of its maximum value. This is because of the high surface/ volume ratio for small bubbles, which, moreover, are uncollided. The maximum presence of walls occurs approximately in the middle of the phase transition, when the fraction of volume is $f_{b} \simeq 0.63$ for the exponential case and $f_{b} \simeq 0.49$ for the simultaneous case. Finally, when only a $1 \%$ of space remains in the false vacuum, the uncollided area is still relatively high. This is more visible in the exponential case, where $\left\langle S_{\text {tot }}\right\rangle$ is more than a $10 \%$ of its maximum value. This relatively high value (compared to $f_{S}$ ) occurs because $f_{S}$ is a fraction of an ever-increasing surface. The main qualitative difference in these curves is that, in the simultaneous-nucleation case, the evolution is quite symmetrical around the midpoint of the transition, while, for the exponential rate, both the variation of $f_{S}$ and the maximum of $\left\langle S_{\text {tot }}\right\rangle$ are shifted towards the end of the transition. This happens because the exponentially growing number of new (smaller) bubbles favors a higher total surface at later times. Nevertheless, in both cases, the maximum of $\left\langle S_{\text {tot }}\right\rangle$ occurs near the time at which $f_{b}=0.5$.

For the delta-function rate, all the bubbles have the same size, and we have $\left\langle S_{\text {tot }}\right\rangle \propto\langle S\rangle$. In contrast, for the exponential rate, the size of a bubble depends on its nucleation time, and the total average area is not proportional to the individual average area. In the left panel of Fig. 10 we show the evolution of the individual average area for this model for bubbles nucleated a different times (we use the natural time unit $\beta^{-1}$, and we show the fraction of surface for reference). We see that the curves of $\langle S\rangle$ are more symmetrical than those of $\left\langle S_{\text {tot }}\right\rangle$. The average fraction of uncollided wall of the bubble, $\exp \left[-I(t)+I\left(t_{N}\right)\right]$, coincides with the probability that a single point on the wall remains uncollided, $P_{u}\left(t, t_{N}\right)$. For the delta-function nucleation rate, we have $t_{N}=t_{*}$ for all bubbles, and we obtain $P_{u}=e^{-I(t)}=P_{f v}(t)$, which is already shown in the right panel of Fig. 9. For the exponential rate, we show this quantity for a few values of $t_{N}$ in the right panel of Fig. 10. The limit $t_{N} \rightarrow-\infty$ gives the volume fraction $P_{f v}(t)=$ $1-f_{b}(t)$ (gray curve). As already mentioned, the variation of this fraction occurs in a time $\Delta t \simeq 6.13 \beta^{-1}$. For a bubble nucleated at $t_{N} \lesssim t_{e}-4 \beta^{-1}$, its uncollided fraction is very close to this curve. Bubbles that nucleate later are initially uncollided, but their uncollided wall fractions fall faster, such that for $t=t_{e}+2 \beta^{-1}$ they are all vanishingly small.

At a given time $t, P_{u}\left(t, t_{N}\right)$ gives the uncollided wall fraction of a bubble of radius $R=v\left(t-t_{N}\right)$. In the left panel of Fig. 11 we show this fraction as a function of $R$ at different times $t$. Very small bubbles (which nucleated very recently) are completely uncollided, while larger bubbles have a fraction of their wall already collided. For a very large bubble (i.e., for $t_{N} \rightarrow-\infty$ ), its uncollided wall fraction is just given by $P_{f v}(t)$. We see that, at early times (black solid line), bubbles of all sizes are almost uncollided, while at later times (gray solid line) the walls of all but the smallest bubbles have completely disappeared.

In this model there is a wide range of bubble sizes, and a quantity of interest is the size distribution $d n / d R=$ $V^{-1} d N / d R$. It is easily obtained using $d N$ from Eq. (58) 

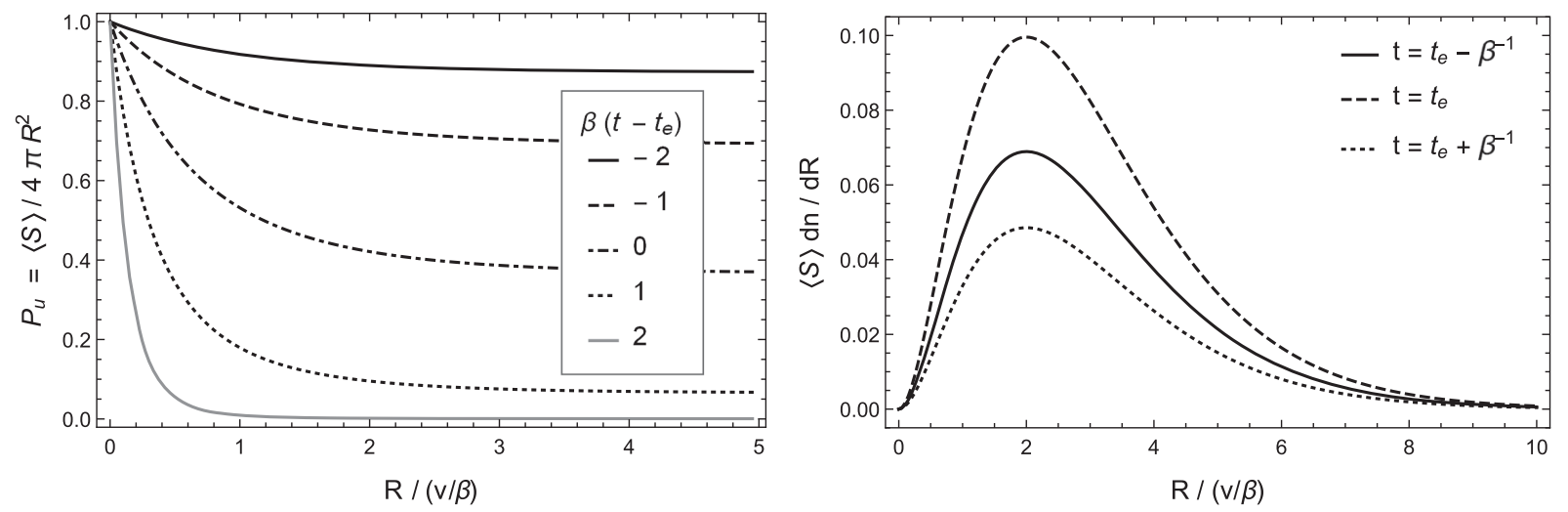

FIG. 11. Uncollided wall fraction of a bubble of radius $R$ (left) and distribution of the total uncollided wall area in bubbles of a different sizes (right).

and $|d R|=v\left(t_{N}\right)\left|d t_{N}\right|$, where, for constant velocity, the nucleation time of a bubble of size $R$ is given by $t_{N}=t-R / v$. We thus have $d n / d R=\bar{\Gamma}(t-R / v) / v$, where $\bar{\Gamma}$ is the average nucleation rate $\Gamma\left(t_{N}\right) P_{f v}\left(t_{N}\right)$. Hence, the size distribution has always the same shape and is only shifted to higher $R$ at later times. For the exponential nucleation rate, the maximum is at $R_{\max }=$ $v\left(t-t_{e}\right)$ and takes the constant value $d n /\left.d R\right|_{\max }=(\beta / v)^{4} /$ $8 \pi e$. Another quantity of interest is the volume-weighted distribution of bubble sizes, $R^{3} d n / d R$, which is associated to the energy that has been released in bubbles of a given size. These quantities have been already discussed in the literature (see, e.g., [1]). The surface-weighted size distribution $R^{2} d n / d R$ may also be of interest, depending on the application. In this case, it may be more appropriate to use the uncollided surface as a weight, $\langle S\rangle d n / d R$, which for this model is given by

$$
\langle S\rangle \frac{d n}{d R}=\frac{1}{2} \frac{\beta^{4}}{v^{4}} I(t) e^{-I(t)} R^{2} e^{-\beta R / v} .
$$

This distribution is shown at three times around $t_{e}$ in the right panel of Fig. 10. We see that it has always the same shape and only changes its amplitude, which is proportional to $\left\langle S_{\text {tot }}(t)\right\rangle$. The maximum is at the fixed value $R=2 v / \beta$. Thus, the normalized distribution

$$
\frac{1}{\left\langle S_{\text {tot }}\right\rangle} \frac{d\left\langle S_{\text {tot }}\right\rangle}{d R}=\frac{\langle S\rangle}{\left\langle S_{\text {tot }}\right\rangle} \frac{d N}{d R}=\frac{1}{2} \frac{\beta^{3}}{v^{3}} R^{2} e^{-\beta R / v}
$$

does not depend on time.

\section{Time correlations in the envelope}

The function $\left\langle S_{\text {tot }}(t)\right\rangle$ describes the turning on and off of the system of walls, which sources several of the consequences of the phase transition. However, as already mentioned, in some cases the relevant quantity will be the time correlation $\left\langle S_{\text {tot }}(t) S_{\text {tot }}\left(t^{\prime}\right)\right\rangle$, or even correlations between individual bubbles or between parts of bubbles. The total surface involves a sum over individual bubbles, $S_{\text {tot }}=\sum_{i} S_{i}$, and we may write

$S_{\mathrm{tot}}(t) S_{\mathrm{tot}}\left(t^{\prime}\right)=\sum_{i} S_{i}(t) S_{i}\left(t^{\prime}\right)+\sum_{i} \sum_{j \neq i} S_{i}(t) S_{j}\left(t^{\prime}\right)$.

Taking the ensemble average, the terms in the first sum involve time correlations of a single bubble. These terms will depend only on the times $t, t^{\prime}$ and on the nucleation time $t_{N}$, but not on the bubble position. Therefore, in a volume $V$, we may evaluate the sum by replacing $\sum_{i} \rightarrow V \int \Gamma\left(t_{N}\right) P_{f v}\left(t_{N}\right) d t_{N}$, like we did in Sec. IV B. On the other hand, the terms in the double sum in Eq. (67) will depend on the bubble separation $l$. Therefore, the sum over $j$ can be replaced by the integral $4 \pi \int d l l^{2}$. The result of this integral does not depend on the bubble positions. Then, the sum over $i$ can be evaluated as before. We shall study these two contributions separately.

Let us first consider a given bubble at two different times $t, t^{\prime}$. The uncollided area at each time is given by Eq. (55), and we have

$$
\begin{aligned}
\left\langle S(t) S\left(t^{\prime}\right)\right\rangle= & R^{\prime 2} R^{2} \int_{0}^{2 \pi} d \phi^{\prime} \int_{0}^{\pi} \sin \theta^{\prime} d \theta^{\prime} \int_{0}^{2 \pi} d \phi \\
& \times \int_{0}^{\pi} \sin \theta d \theta\left\langle 1_{S(t)}(\theta, \phi) 1_{S\left(t^{\prime}\right)}\left(\theta^{\prime}, \phi^{\prime}\right)\right\rangle .
\end{aligned}
$$

The angles correspond to directions $\hat{r}, \hat{r}^{\prime}$ indicating points $p, p^{\prime}$ on the surfaces $S(t)$ and $S\left(t^{\prime}\right)$, respectively. For each pair $\hat{r}, \hat{r}^{\prime}$, the product $1_{S(t)} 1_{S\left(t^{\prime}\right)}$ takes the value 0 or 1 , the latter with probability $P_{\hat{r}, \hat{r}^{\prime}}(1)=P_{p, p^{\prime}}^{S}\left(t, t^{\prime}, t_{N}, s\right)$ given by Eq. (28). This probability depends on a single angle, namely, between $\hat{r}$ and $\hat{r}^{\prime}$. Using the relation (30) for the point separation $s$ as a function of the angle between $\hat{r}$ and $\hat{r}^{\prime}$, we obtain 

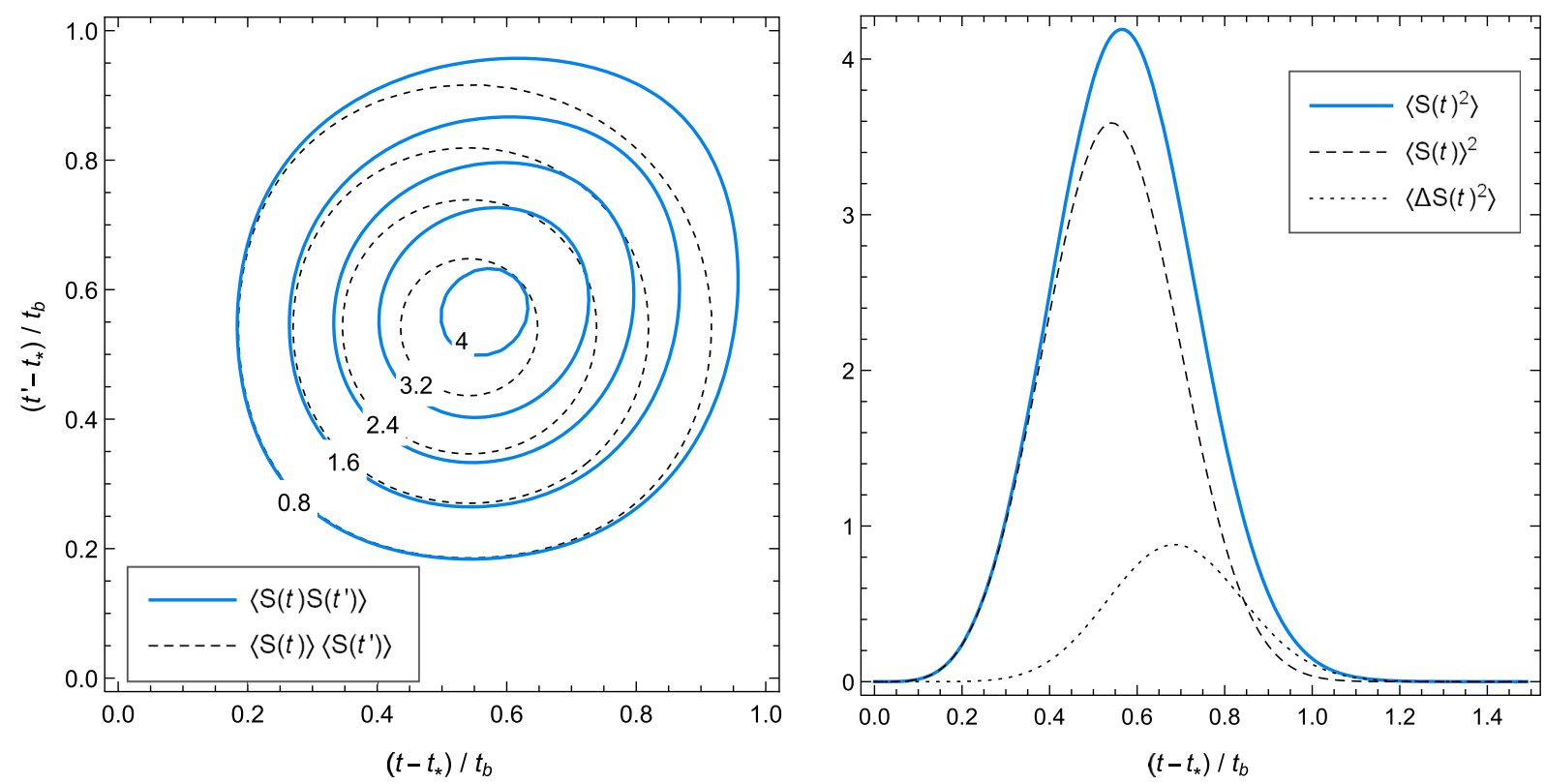

FIG. 12. Surface correlation of a bubble between two times for a delta-function rate, in units of $d_{b}^{4}$. Left panel: contours of $\left\langle S(t) S\left(t^{\prime}\right)\right\rangle$ and $\langle S(t)\rangle\left\langle S\left(t^{\prime}\right)\right\rangle$. Right panel: the equal-time values and their difference.

$\left\langle S(t) S\left(t^{\prime}\right)\right\rangle=8 \pi^{2} R R^{\prime} e^{-I(t)-I\left(t^{\prime}\right)+I\left(t_{N}\right)} \int_{R^{\prime}-R}^{R^{\prime}+R} d s s e^{I_{n}\left(t, t^{\prime}, s\right)}$,

where $I_{\cap}$ is given by Eq. (15). If we consider two different bubbles, whose centers are a distance $l$ apart, we may repeat the same steps which lead to Eq. (69). The only difference is that now we have two surfaces belonging to different bubbles, so we replace $S\left(t^{\prime}\right)$ with $S^{\prime}\left(t^{\prime}\right)$ in Eq. (68), and the probability $P_{\hat{r}, \hat{r}^{\prime}}(1)$ is given by Eqs. (50)-(51). We thus have

$$
\begin{aligned}
& \left\langle 1_{S(t)}(\theta, \phi) 1_{S^{\prime}\left(t^{\prime}\right)}\left(\theta^{\prime}, \phi^{\prime}\right)\right\rangle \\
& \quad=P_{p, p^{\prime}}^{S S^{\prime}}\left(t, t^{\prime}, t_{N}, t_{N}^{\prime}, S\right) \Theta\left(d-R\left(t_{N}^{\prime}, t\right)\right) \Theta\left(d^{\prime}-R\left(t_{N}, t^{\prime}\right)\right) .
\end{aligned}
$$

We obtain

$$
\begin{aligned}
\left\langle S(t) S^{\prime}\left(t^{\prime}\right)\right\rangle= & 2 \pi R^{2} R^{\prime 2} e^{-I(t)-I\left(t^{\prime}\right)+I\left(t_{N}\right)+I\left(t_{N}^{\prime}\right)} \int_{0}^{2 \pi} d \phi \int_{0}^{\pi} d \theta \sin \theta \int_{0}^{\pi} d \theta^{\prime} \sin \theta^{\prime} \\
& \times \exp \left[I_{\cap}\left(t, t^{\prime}, s\right)\right] \Theta\left(d-R\left(t_{N}^{\prime}, t\right)\right) \Theta\left(d^{\prime}-R\left(t_{N}, t^{\prime}\right)\right) .
\end{aligned}
$$

We could also change the variables of integration from the angles to the distances $s, d, d^{\prime}$ through Eqs. (44)-(45). The result depends on the bubble separation $l$.

\section{Simultaneous nucleation}

For a delta-function rate we have $t_{N}=t_{*}, I\left(t_{N}\right)=0$, and $I_{\cap}=n_{b} V_{n}$, with

$V_{\cap}=\frac{\pi}{12}\left(R+R^{\prime}-s\right)^{2}\left[s+2\left(R+R^{\prime}\right)-\frac{3\left(R^{\prime}-R\right)^{2}}{s}\right]$.

In the two-bubble case, we have also $t_{N}^{\prime}=t_{*}$ and $I\left(t_{N}^{\prime}\right)=0$. Besides, the Heaviside functions become $\Theta(d-R) \Theta\left(d^{\prime}-R^{\prime}\right)$. At equal times we have $R^{\prime}=R$, and the expressions become simpler. In particular, we have

$$
V_{\cap}=\frac{\pi}{12}(2 R-s)^{2}(s+4 R)
$$

In any case, the integrals in Eqs. (69) and (71) must be computed numerically.

In Figs. 12 and 13 we consider the surface correlations for a single bubble. In Fig. 12 we compare the functions $\left\langle S(t) S\left(t^{\prime}\right)\right\rangle$ and $\langle S(t)\rangle\left\langle S\left(t^{\prime}\right)\right\rangle$. The latter has a quite simpler expression, since we have (in this model) $\langle S\rangle=4 \pi R^{2} e^{-I}$, and could be used as an approximation for the former. Such an approximation corresponds to assuming that the two surfaces are uncorrelated. We see that these quantities are quite similar. In particular, the approximation is very good initially (i.e., for small values of $t-t_{*}$ and $t^{\prime}-t_{*}$ ). However, they depart at later times, where the uncorrelated 

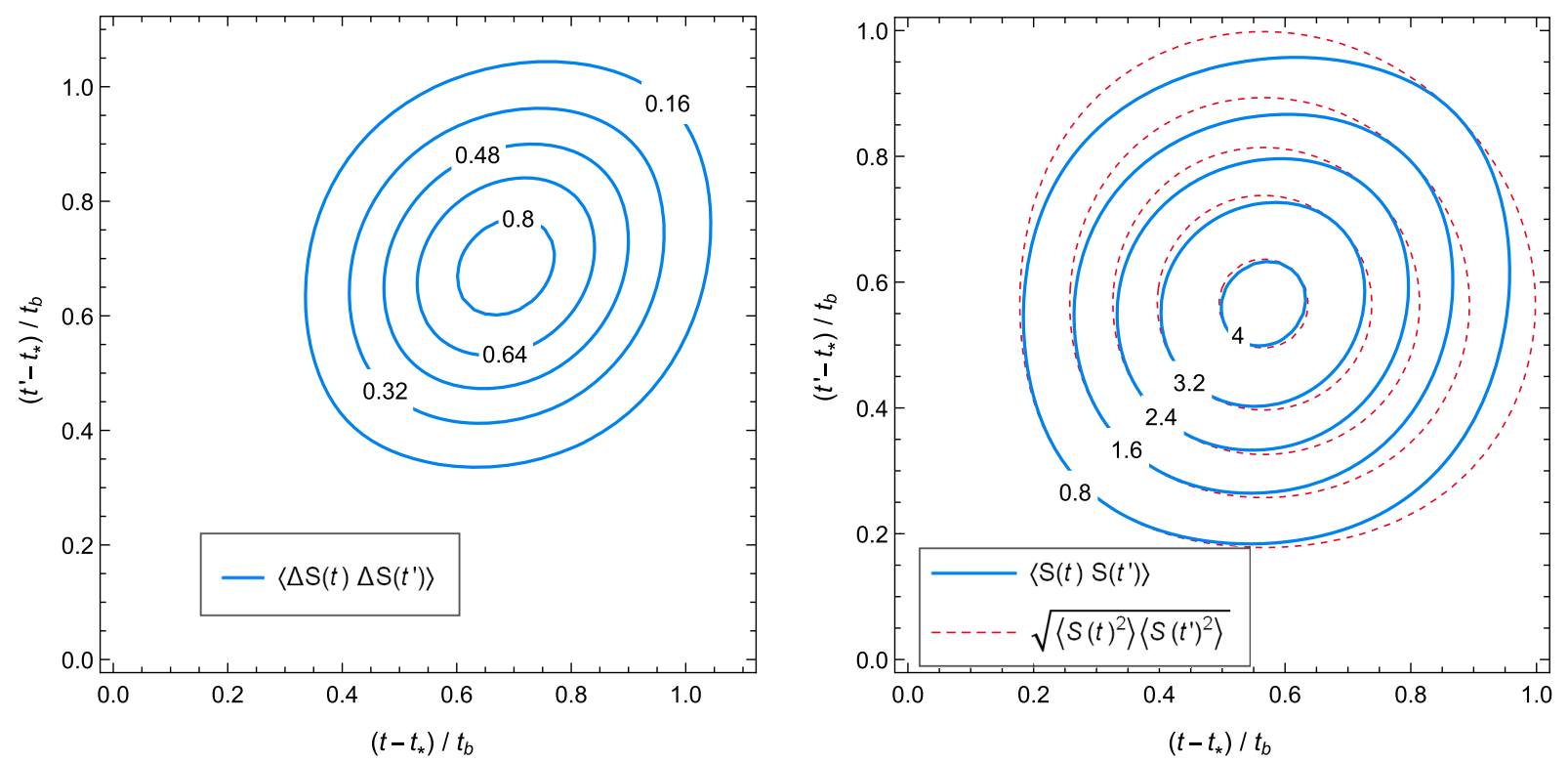

FIG. 13. Surface correlation of a bubble between different times for a delta function rate. Left panel: contours of $\left\langle\Delta S(t) \Delta S\left(t^{\prime}\right)\right\rangle$. Right panel: contours of $\left\langle S(t) S\left(t^{\prime}\right)\right\rangle$ and $\sqrt{\left\langle S(t)^{2}\right\rangle\left\langle S\left(t^{\prime}\right)^{2}\right\rangle}$. The areas are in units of $d_{b}^{2}$.
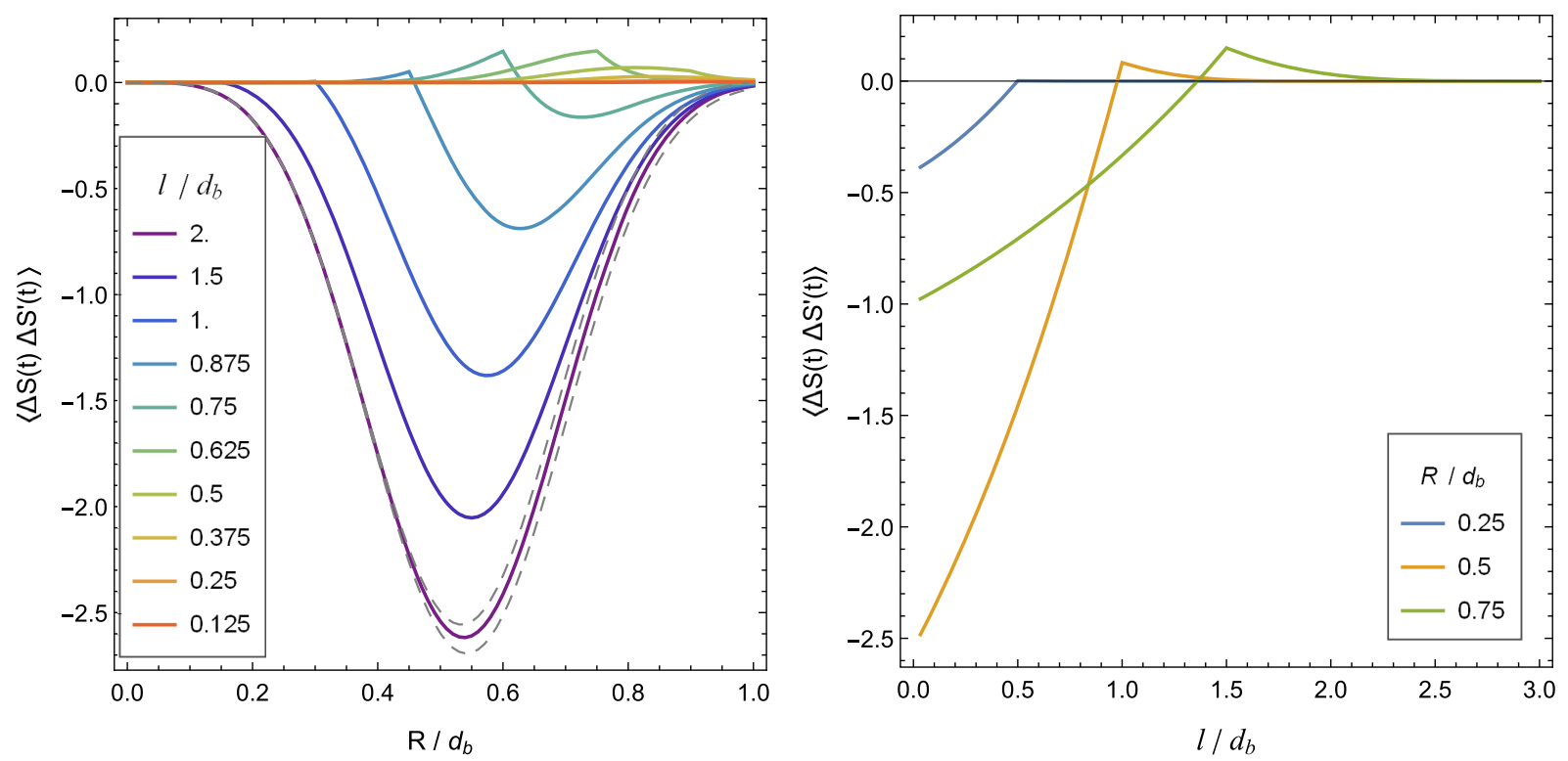

FIG. 14. The equal-time function $\left\langle\Delta S(t) \Delta S^{\prime}(t)\right\rangle$ for a delta-function rate (in units of $d_{b}^{4}$ ) for two bubbles separated a distance $l$, as a function of the bubble radius (left panel) and as a function of the bubble separation (right panel). The dashed curves in the left panel correspond to the functions $\left\langle S(t)^{2}\right\rangle / 4-\langle S(t)\rangle^{2}$ (upper curve) and $-3\langle S(t)\rangle^{2} / 4$ (lower curve).

function tends to zero more rapidly. In the right panel, the variance $\left\langle\Delta S^{2}\right\rangle$, where $\Delta S=S-\langle S\rangle$, is also shown. The left panel of Fig. 13 shows the covariance $\left\langle\Delta S(t) \Delta S\left(t^{\prime}\right)\right\rangle$. In the right panel we compare the function $\left\langle S(t) S\left(t^{\prime}\right)\right\rangle$ with $\sqrt{\left\langle S(t)^{2}\right\rangle\left\langle S\left(t^{\prime}\right)^{2}\right\rangle}$. The latter is the result we would obtain if $S(t)$ and $S\left(t^{\prime}\right)$ were maximally correlated. Since Eqs. (69)-(72) are simpler for equal times, this function can also be used as an approximation for the former. By definition, both coincide at $t=t^{\prime}$, so this approximation is better than the uncorrelated one at later times. On the other hand, it deviates for large $\left|t-t^{\prime}\right|$.

In Fig. 14 we show the result for two bubbles separated a distance $l$. We consider the deviations $\Delta S$, $\Delta S^{\prime}$, and we plot only the equal-time case. In the left 

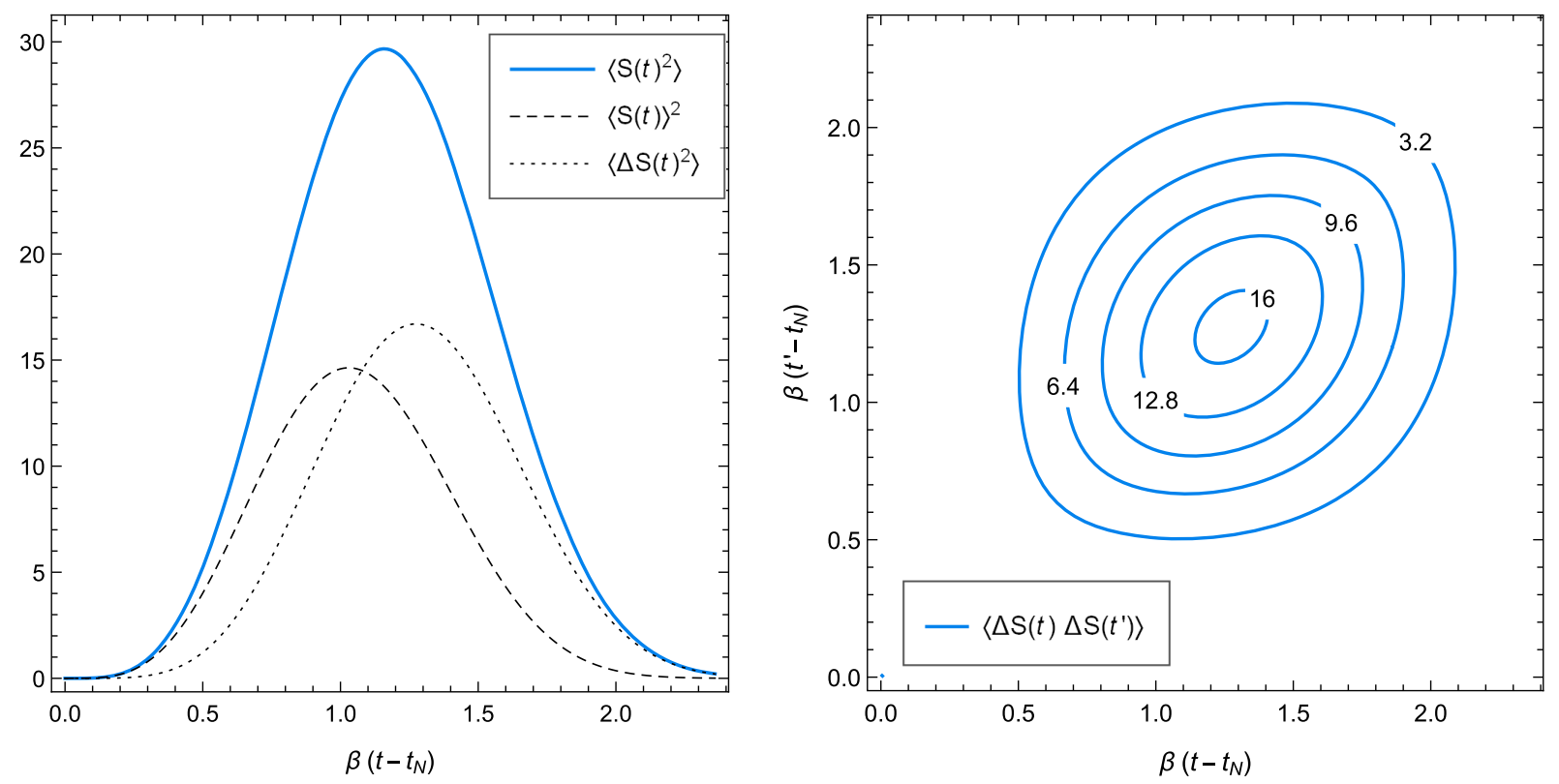

FIG. 15. Surface correlation in the case of an exponential nucleation rate, for a bubble nucleated at time $t_{N}=t_{\text {med }}$ such that $f_{b}\left(t_{\text {med }}\right)=0.5$. The area is in units of $(v / \beta)^{2}$. Left panel: equal-time values of $\left\langle S(t) S\left(t^{\prime}\right)\right\rangle,\langle S(t)\rangle\left\langle S\left(t^{\prime}\right)\right\rangle$, and $\left\langle\Delta S(t) \Delta S\left(t^{\prime}\right)\right\rangle$. Right panel: contours of $\left\langle\Delta S(t) \Delta S\left(t^{\prime}\right)\right\rangle$.

panel, the covariance ${ }^{17}\left\langle\Delta S(t) \Delta S^{\prime}(t)\right\rangle$ is plotted as a function of the bubble radius $R$, or, equivalently, as a function of time, since we have $R / d_{b}=\left(t-t_{*}\right) / t_{b}$. The curves of different colors correspond to various values of the bubble separation $l$. We see that the correlation vanishes for large $l$, i.e., we have $\left\langle S(t) S^{\prime}(t)\right\rangle \rightarrow\langle S(t)\rangle\langle S(t)\rangle$ for $l \rightarrow \infty$. On the other hand, the maximal correlation is attained for $l \rightarrow 0$. This is also appreciated in the right panel, which shows the covariance as a function of $l$ for a few values of $R$. In all these curves, there is a sudden change in the behavior at the point $l=2 R$, i.e., when the two bubbles come into contact. For $l<2 R$, the two bubbles overlap, so a part of their surface is surely collided. One could expect that for $l \rightarrow 0$ the quantity $\left\langle S(t) S^{\prime}(t)\right\rangle$ would match the value $\langle S(t) S(t)\rangle$. However, in this limit, half of each bubble is surely collided, so a better guess would be $\left\langle S(t)^{2}\right\rangle / 4$. This value is indicated by the upper dashed line in the left panel of Fig. 14. We see that this curve does not coincide with the limit $l=0$ for different bubbles. The lower dashed line corresponds to the approximation $\langle S(t)\rangle^{2} / 4$.

\section{Exponential nucleation}

For the exponential nucleation rate, the general characteristics are qualitatively similar to the delta-function rate, as already seen for $\left\langle S_{\text {tot }}\right\rangle$. Let us consider, for instance, the

\footnotetext{
${ }^{17}$ In the general case we have $\left\langle\Delta S(t) \Delta S^{\prime}\left(t^{\prime}\right)\right\rangle=\left\langle S(t) S^{\prime}\left(t^{\prime}\right)\right\rangle-$ $\langle S(t)\rangle\left\langle S^{\prime}\left(t^{\prime}\right)\right\rangle$. Notice that $\left\langle S^{\prime}\left(t^{\prime}\right)\right\rangle=\left\langle S\left(t^{\prime}\right)\right\rangle$. Thus, for equal times we have $\left\langle\Delta S(t) \Delta S^{\prime}(t)\right\rangle=\left\langle S(t) S^{\prime}(t)\right\rangle-\langle S(t)\rangle^{2}$.
}

wall area correlation for an individual bubble, which is given by Eq. (69). For the integral (15) we obtain ${ }^{18}$

$$
I_{\cap}\left(t, t^{\prime}, s\right)=\frac{1}{4} I\left(\frac{t+t^{\prime}}{2}\right) \exp \left(-\frac{\beta s}{2 v}\right)\left[4+\frac{\beta s}{v}-\frac{\left[\beta\left(t-t^{\prime}\right)\right]^{2}}{\beta s / v}\right]
$$

The final integration with respect to $s$ in Eq. (69) must be done numerically. In Fig. 15 we show the surface correlation functions $\left\langle S(t) S\left(t^{\prime}\right)\right\rangle, \quad\langle S(t)\rangle\left\langle S\left(t^{\prime}\right)\right\rangle$, and $\left\langle\Delta S(t) \Delta S\left(t^{\prime}\right)\right\rangle$. The result depends on the nucleation time $t_{N}$, and we consider a bubble nucleated when $f_{b}=0.5$, i.e., at the time $t_{\text {med }}=t_{e}+\beta^{-1} \log \log 2$. The main difference with other nucleation times is the height of the curves, since older bubbles are larger (see the left panel of Fig. 10). Notice also that the area is measured in the natural units $(v / \beta)^{2}$; in units of the distance $d=v \Delta t$, the values are smaller. The shapes of the equal-time curves (left panel) are qualitatively similar to those of the simultaneous nucleation case. The main quantitative difference is that the value of $\langle S(t)\rangle^{2}$ is not so close to $\left\langle S(t)^{2}\right\rangle$ in this case. The right panel shows the contour plots of $\left\langle\Delta S(t) \Delta S\left(t^{\prime}\right)\right\rangle$, which are also qualitatively similar to the simultaneous case.

\footnotetext{
${ }^{18} \mathrm{We}$ must use the expression (32) for the function $V_{\mathrm{n}}$, where only $r+r^{\prime}=v\left(t+t^{\prime}-2 t^{\prime \prime}\right)$ depends on the integration variable $t^{\prime \prime}$. The Heaviside function $\Theta\left(r+r^{\prime}-s\right)$ gives the condition $t^{\prime \prime}<\left(t+t^{\prime}\right) / 2-s / 2 v$. This bound is always less than the limit of integration $t^{\prime}$ in Eq. (15) because we always have $s>v\left(t-t^{\prime}\right)$.
} 

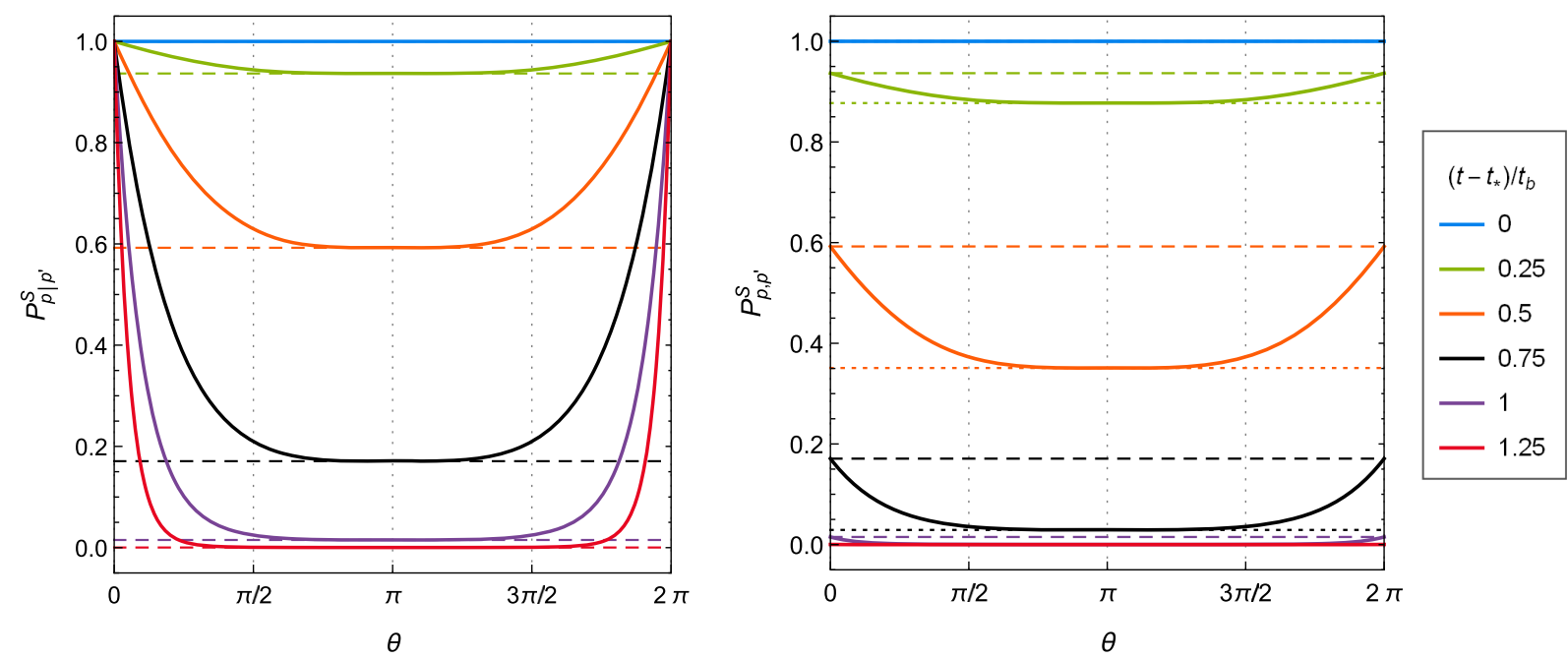

FIG. 16. Conditional probability (left) and joint probability (right) for two points on a bubble wall to be uncollided in the case of simultaneous nucleation, as a function of the separation angle. The horizontal dashed lines indicate the value of $P_{u}(t)=P_{f v}(t)$. The horizontal dotted lines indicate the value $P_{u}(t)^{2}$.

\section{Correlation between different parts of a bubble wall}

We shall now consider the probability for two points on a given bubble wall to be uncollided at a given time $t$. We must take $t^{\prime}=t$ in Eqs. (27)-(32). In this case we have $s=R \sqrt{2(1-\cos \theta)}=2 R \sin (\theta / 2)$. The conditional probability that a point $p$ is uncollided assuming that another point $p^{\prime}$ is uncollided is given by Eq. (27). For simultaneous nucleation, we have $I_{\cap}=n_{b} V_{n}$, with $V_{n}$ given by Eq. (73), and we obtain

$$
\begin{aligned}
P_{p \mid p^{\prime}}^{S}(t, \theta)= & \exp \left[-\frac{4 \pi}{3}\left(\frac{t-t_{*}}{\Delta t_{b}}\right)^{3}\right. \\
& \left.\times\left(\frac{3}{2} \sin (\theta / 2)-\frac{1}{2} \sin ^{3}(\theta / 2)\right)\right] .
\end{aligned}
$$

For the exponential case, $I_{\cap}$ is given by Eq. (74), and at equal times we obtain

$$
\begin{aligned}
P_{p \mid p^{\prime}}^{S}\left(t, t_{N}, \theta\right)= & \exp \left\{e ^ { \beta ( t - t _ { * } ) } \left[e^{-\beta\left(t-t_{N}\right) \sin (\theta / 2)}\right.\right. \\
& \left.\left.\times\left(1+\frac{1}{2} \beta\left(t-t_{N}\right) \sin (\theta / 2)\right)-1\right]\right\} .
\end{aligned}
$$

The joint probability that both $p$ and $p^{\prime}$ are in the false vacuum is given by Eq. (28), $P_{p, p^{\prime}}^{S}=e^{-I(t)+I\left(t_{N}\right)} P_{p \mid p^{\prime}}$ (for the simultaneous case, the prefactor is just $\left.e^{-I(t)}\right)$.

In Fig. 16 we show the result for simultaneous nucleation. At the beginning of the phase transition we have $P_{p, p^{\prime}}^{S}=P_{p \mid p^{\prime}}^{S}=1$, since the two points are uncollided because the whole bubble is isolated. By the end of the phase transition, the probability that both points are uncollided vanishes unless we assume that one of them is uncollided. In this case (left panel), for $\theta \simeq 0$ the probability will never vanish. At intermediate times, assuming that $p^{\prime}$ is uncollided, the probability that $p$ is also uncollided falls with the distance from $p^{\prime}$. For $\theta=\pi$ Eq. (75) gives the value $P_{u}(t)$, which is the probability for an arbitrary point. This indicates that the correlation is lost exactly at the antipodal point. This happens because in this model all the bubbles have the same radius $R$, and the correlation must vanish beyond a distance $2 R$. Indeed, for both points $p$ and $p^{\prime}$ to be affected by the same bubble, their separation must be smaller than $2 R$. On the other hand, we see that the limit $P_{p \mid p^{\prime}}^{S}=P_{u}$ is approached already for $\theta \gtrsim \pi / 2$. The behavior of the joint probability is similar, except that it takes the single-point value $P_{u}(t)$ for $\theta=0$, while for $\theta=\pi$ it takes the value $P_{u}(t)^{2}$, corresponding to independent variables.

In Fig. 17 we show the behavior of the conditional probability for the exponential case (the joint probability has a simple relation with the latter, like in the simultaneous case). In the present case, $P_{p \mid p^{\prime}}^{S}$ depends on the nucleation time $t_{N}$. We consider two different nucleation times $t_{N}$ in each panel of Fig. 17, and we show the probability $P_{p \mid p^{\prime}}^{S}(\theta)$ at different times $t$ like we did for the simultaneous case. The main difference with that case is the fact that, at the maximum distance $(\theta=\pi)$, the probability does not take the single-point value $P_{u}\left(t, t_{N}\right)$ but a higher value, $P_{u}\left(t, t_{N}\right) \exp \left[I\left(t_{N}\right) \beta\left(t-t_{N}\right) / 2\right]$. This means that the point $p$ is never independent of the point $p^{\prime}$ : if $p^{\prime}$ is uncollided, the probability of $p$ being also uncollided is always higher than without this condition. The value $P_{u}\left(t, t_{N}\right)$ can only be reached in the trivial limit $t \rightarrow t_{N}$ (i.e., if the bubble has just nucleated), and in the limit $t_{N} \rightarrow-\infty$.

In the left panel of Fig. 17 we considered a relatively early nucleation time, $t_{N}=t_{e}-4 \beta^{-1}$, and times $t$ up to 

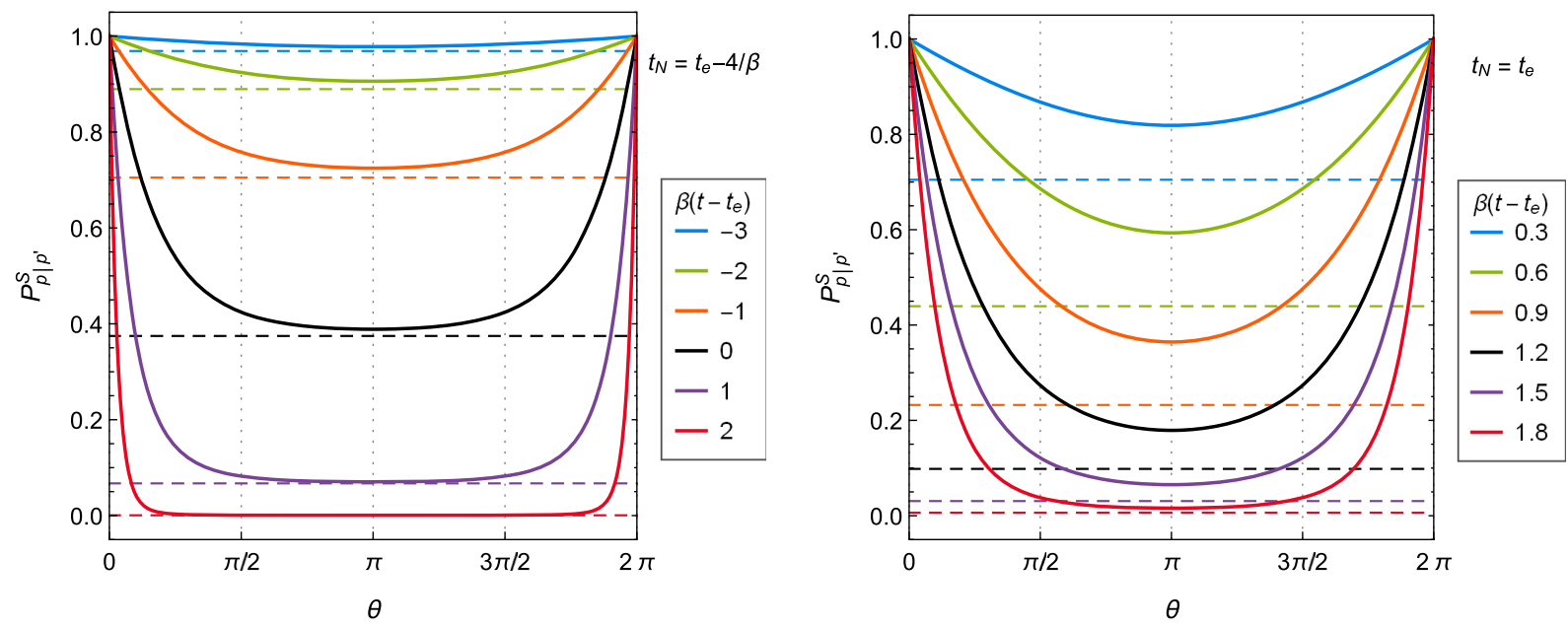

FIG. 17. Conditional probability as a function of the separation angle in the exponential case, at different times $t$ for a bubble nucleated at $t_{N}=t_{e}-4 \beta^{-1}$ (left) and at $t_{N}=t_{e}$ (right). The horizontal dashed lines indicate the value of $P_{u}\left(t, t_{N}\right)=P_{f v}(t) / P_{f v}\left(t_{N}\right)$.

$t_{e}+2 \beta^{-1}$ (at which the phase transition is already completed, as can be seen, e.g., in Fig. 10). We see that the result is qualitatively similar to the simultaneous case: although the probability never takes the single-point value $P_{u}\left(t, t_{N}\right)$, all the curves approach this value for $\theta \simeq \pi$. However, a bubble nucleated so early is not representative, since bubbles which nucleate later are much more abundant. In the right panel of Fig. 17 we consider a bubble nucleated at $t_{N}=t_{e}$, at which the maximum nucleation of bubbles occurs, and which is close to the time $t_{\text {med }}$ at which $f_{b}=0.5$. In this case, the correlation between the points $p$ and $p^{\prime}$ is never lost. This happens because we have a wide range of bubble sizes. Therefore, two points on the wall of a bubble of radius $R$ may be both affected by a single bubble of radius larger than $R$. Only in the case of a very large bubble (nucleated at $t_{N} \ll t_{e}$ ) this does not hold, since two points on its surface can only be affected by bubbles of smaller radii. In such a case the point correlation decreases significantly with the separation, as observed in the left panel.

\section{APPLICATIONS TO COSMOLOGICAL CONSEQUENCES}

We shall now discuss how some of the quantities derived in previous sections enter the consequences of the phase transition.

\section{A. Electroweak baryogenesis}

In the electroweak phase transition, the violation of baryon number takes place in the symmetric phase outside the bubbles and is biased by net charge densities generated in front of the moving walls. The resulting baryon number density $n_{B}$ depends on the wall velocity. For very slow velocities, the plasma will be near equilibrium, and the net baryon number will vanish. On the other hand, for very high velocities (close to the speed of sound), the processes which violate baryon number (sphalerons) will have no time to generate a significant amount of baryons as the wall passes. As a consequence, there is a maximum baryon generation at a velocity in the range $10^{-3}-10^{-1}$ (see [42] for a recent discussion). In most computations of electroweak baryogenesis for specific models, the global dynamics of the phase transition is not taken into account. In particular, the wall velocity is estimated at the "onset" of nucleation (i.e., at the instant at which there is a bubble per Hubble volume). However, for velocities in the above range, which correspond to deflagrations, there will be a reheating in the symmetric phase, which will cause the wall velocity to decrease from the initial value.

For the small velocities which are favorable for baryogenesis, a homogeneous reheating can be assumed $[38,40,43]$. The time-temperature relation for this case was derived in Ref. [44]. The expression for $T(t)$ contains a term that decreases with the scale factor (accounting for the adiabatic cooling) and a term proportional to the fraction of volume occupied by bubbles, $f_{b}$ (accounting for the reheating). In many scenarios there is little supercooling, $\left(T_{c}-T\right) \ll T_{c}$ [45], and we can linearize quantities that vanish at $T=T_{c}$, except for those with a rapid variation, such as $\Gamma$ or $f_{b}$. Thus, e.g., the adiabatic cooling relation becomes $T_{c}-T=T_{c} H\left(t-t_{c}\right)$, but the release of latent heat introduces a term proportional to $L f_{b}(t)$, where $L$ is the latent heat. With these approximations, analytic expressions for the development of the phase transition were obtained in Ref. [46]. Due to the high sensitivity of the nucleation rate with the temperature, a simultaneous nucleation at a certain time $t_{*}$ (corresponding to the minimum temperature reached $T_{*}$ ) is a good approximation. The subsequent evolution depends on the parameter

$$
q \simeq \frac{L}{\rho_{R}\left(T_{c}\right)-\rho_{R}\left(T_{*}\right)},
$$



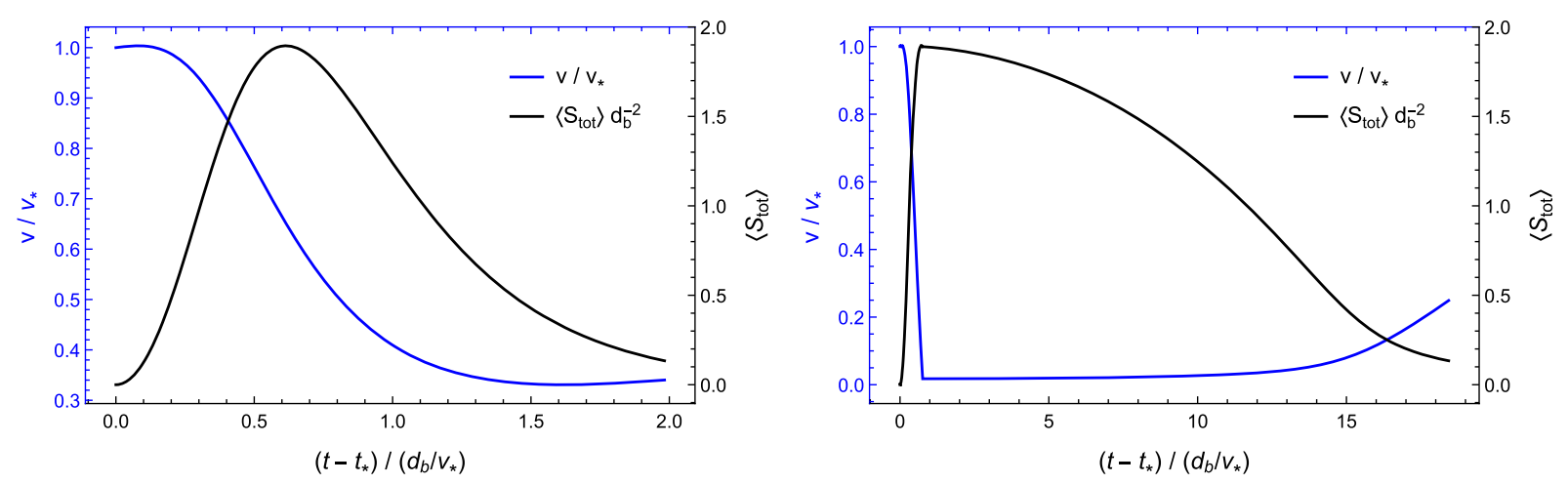

FIG. 18. The wall velocity and the average wall area for $q=0.8$ (left) and $q=2$ (right).

where $\rho_{R}(T)$ is the energy density in radiation. Thus, $q$ gives the ratio of the released energy to the energy which is needed to reheat the plasma back to $T_{c}$. This parameter is important since the wall velocity vanishes at $T=T_{c}$. In particular, the approximation $v \propto T_{c}-T$ leads to

$$
\frac{v}{v_{*}}=1-q f_{b}(t)+\frac{t-t_{*}}{t_{*}-t_{c}} .
$$

Since the nucleation is simultaneous, we have $f_{b}=1-e^{-I}$, with $I=\frac{4 \pi}{3}\left(R / d_{b}\right)^{3}$.

There are two well-differentiated behaviors depending on whether the value of $q$ is greater or less than 1 [46]. For $q<1$ the function $R(t)$ can be approximated by the relation

$$
\frac{t-t_{*}}{d_{b} / v_{*}}=\frac{R}{d_{b}}+\frac{\pi q}{3}\left(\frac{R}{d_{b}}\right)^{4}
$$

On the other hand, for $q>1$, the reheating takes the temperature very close $T_{c}$, and the wall velocity may decrease by a few orders of magnitude. As a consequence, after an initial reheating stage in which the approximation (79) is valid, a relatively long stage of very slow growth is established. Assuming an approximate phase equilibrium during this second stage, the evolution can be obtained by equating the released energy density $L\left\langle S_{\text {tot }}\right\rangle v d t$ to the decrease of energy density due to the expansion, $4 \rho_{R} H d t$ (since $\rho_{R} \propto T^{4}$ and $\dot{T}=-H T$ ). This gives

$$
\frac{v}{v_{*}}=\frac{d_{b} / v_{*}}{t_{*}-t_{c}} \frac{e^{I}}{q 4 \pi\left(R / d_{b}\right)^{2}} .
$$

Here, the relations $\left\langle S_{\text {tot }}\right\rangle=\langle S\rangle n_{b}, \rho_{R}\left(T_{c}\right)-\rho_{R}\left(T_{*}\right) \simeq$ $4 \rho_{R}\left(T_{c}-T_{*}\right) / T_{c}$, and $\left(T_{c}-T_{*}\right)=T_{c} H\left(t_{*}-t_{c}\right)$ have been used. An approximation for the relation $R(t)$ in this slow stage is given in [46],

$$
\frac{t-t_{*}}{d_{b} / v_{*}}=\frac{t_{*}-t_{c}}{d_{b} / v_{*}}\left(q f_{b}-1\right)+\frac{e^{I}}{q 4 \pi\left(R / d_{b}\right)^{2}} .
$$

The evolution of the wall velocity for these approximations is shown in Fig. 18 for the two cases $q<1$ and $q>1$, together with the average uncollided surface. For the case $q<1$ we considered a relatively high value $q=0.8$, so that we obtain a velocity variation of order 1 . The time $t_{*}-t_{c}$ depends on the supercooling parameter $\left(T_{c}-T_{*}\right) / T_{c}$. This time is usually an order of magnitude greater than the timescale of the reheating, which is given by $d_{b} / v_{*}$. Therefore, we used the value $\left(t-t_{*}\right) /\left(d_{b} / v_{*}\right)=15$. For the case $q>1$ we considered the same supercooling parameter, and we chose $q=2$. For $q \gg 1$ the effect will be more pronounced.

The velocity decrease may suppress or enhance the baryon generation, depending on whether the initial velocity $v_{*}$ is smaller or higher than the maximum of $n_{B}$ $[38,40,43]$. If the velocity variation occurs around the maximum, then it will not cause a significant effect on baryogenesis. Therefore, we shall consider only the cases in which the velocity variation occurs entirely on the left or on the right of the maximum of $n_{B}(v)$. For simplicity, we shall assume that in these cases the dependence is either $n_{B} \propto v$ or $n_{B} \propto v^{-1}$ (for a motivation of this dependence and analytic approximations for the whole velocity range, see $[40,43])$. If the baryon number density generated at the wall is of the form $n_{B} \propto v$, the total baryon number produced will be suppressed with respect to the initial value $n_{B *}$. In Fig. 18 we see that the average wall area, which acts as a weight, emphasizes this effect, since $\left\langle S_{\text {tot }}\right\rangle$ is maximal when the baryon density has already begun to decrease.

In the case $n_{B} \propto v^{-1}$ the total baryon density is enhanced with respect to $n_{B *}$. Let us consider this case in more detail, since it is more interesting. We may write $n_{B}(v)=n_{B *} v_{*} / v$. The total baryon number produced at time $t$ in a volume $V$ is given by

$$
V d B=n_{B}(v(t))\left\langle S_{\mathrm{tot}}(t)\right\rangle v(t) d t .
$$

The expression is more clear in terms of the bubble radius,

$$
d B=n_{B}(v)\langle S\rangle d R / d_{b}^{3},
$$

with $\langle S\rangle=4 \pi R^{2} e^{-\frac{4 \pi}{3}\left(R / d_{b}\right)^{3}}$. Thus, in units of $d_{b}$, we have $\int_{0}^{\infty}\langle S\rangle d R=1$. In Fig. 19 we show the local baryon number 

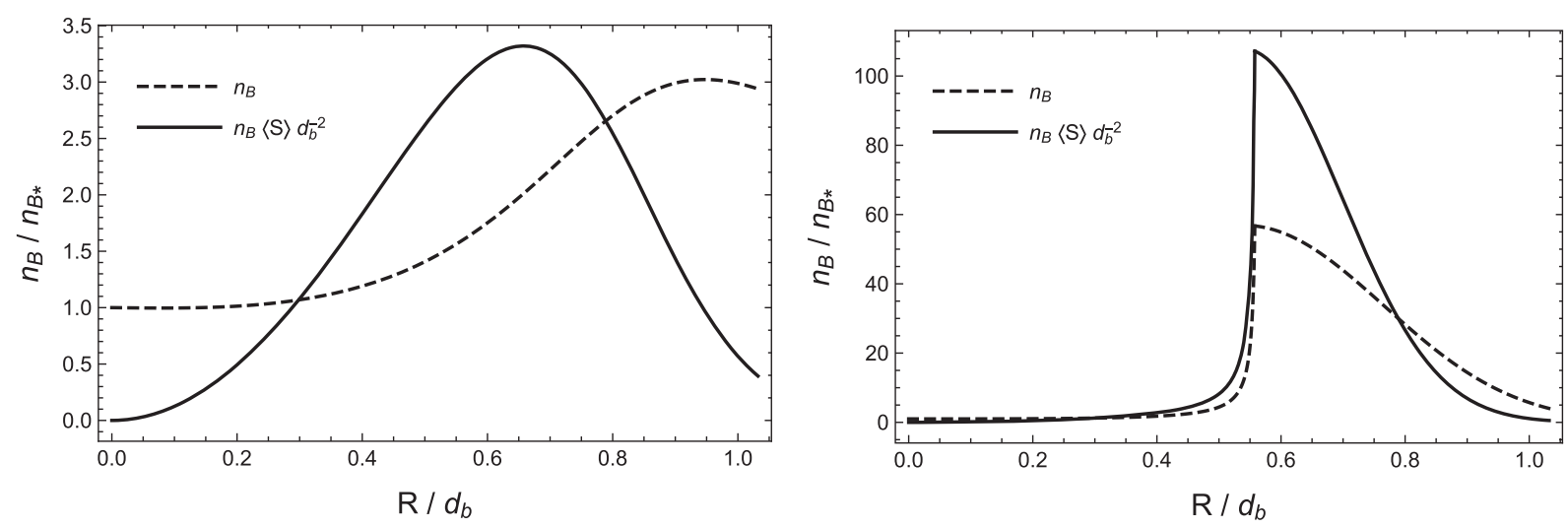

FIG. 19. The baryon number density as a function of the bubble radius for the cases of Fig. 18.

density $n_{B}$ as a function of $R$, together with the weighted density $n_{B}\langle S\rangle$. The function $n_{b}(R)$ gives the profile of the baryon inhomogeneities produced inside each bubble, which may also be of interest, while the product $n_{B}\langle S\rangle$ gives, upon integration, the average baryon number density $B$. For $q<1$, the weight function peaks somewhere between the minimum and maximum value of $n_{B}$. On the other hand, for $q>1$, the highest values of $n_{B}$ have a higher weight. This can be inferred already from Fig. 18. In these specific examples, the total amplification is $B / n_{B *} \simeq 1.74$ for the case $q=0.8$ and $B / n_{B *}=21.2$ for the case $q=2$.

\section{B. Gravitational waves}

As mentioned in the Introduction, the moving walls cause different phenomena that generate GWs, such as sound waves and turbulence, and the walls themselves are a direct source of gravitational radiation. Besides the well-known bubble-collision mechanism, other kinds of deformations from the spherical shape may produce GWs. The exponential growth of wall corrugations due to hydrodynamic instabilities [47-50] may constitute an important example. The relevant quantity in the calculation of gravitational waves is the transverse and traceless projection of the energy-momentum tensor of the source, $\Pi_{i j}(t, \mathbf{k})$ in Fourier space. The energy density per logarithmic wave number of GWs can be written in the form $[31,32]$

$$
\frac{d \rho_{G W}}{d \ln k}=\frac{2 G k^{3}}{\pi} \int_{-\infty}^{\infty} d t \int_{-\infty}^{\infty} d t^{\prime} e^{i k\left(t-t^{\prime}\right)} \Pi\left(t, t^{\prime}, k\right),
$$

where the quantity $\Pi$ is essentially the unequal-time correlator $\left\langle\Pi_{i j} \Pi_{i j}^{*}\right\rangle$, after subtracting a delta function of the wave vector. Below we shall relate the general characteristics of the spectrum of GWs originated at the bubble walls with the average wall area and the its correlations.

Some general properties of the GW spectrum from phase transitions were discussed in Ref. [51]. For colliding bubbles, a statistical argument was used to motivate a source correlator of the form (with separated variables $t, t^{\prime}$ )

$$
\Pi\left(t, t^{\prime}, k\right)=\frac{2}{(2 \pi)^{3}} \rho_{s}^{2} \frac{N}{V} f(k, t) f\left(k, t^{\prime}\right)
$$

where the constant $\rho_{s}$ is the energy density of the source and $N / V$ is the density of collision events. This gives, according to Eq. (84),

$\frac{d \rho_{G W}}{d \ln k}=\frac{4 G \rho_{s}^{2}}{\pi(2 \pi)^{3}} \frac{N}{V} k^{3}\left|\int_{-\infty}^{\infty} d t e^{i k t} f(k, t)\right|^{2} \equiv C k^{3}|\hat{f}(k, k)|^{2}$.

In Ref. [51], the function $f(k, t)$ was further assumed to be of the form

$$
f(k, t)=g(t) F(k)
$$

where the function $g(t)$ vanishes outside a time interval $\left[t_{*}, t_{*}+\Delta t\right]$ in which the phase transition occurs. Here, $t_{*}$ is the initial time and $\Delta t$ is the duration of the phase transition. They considered several examples for this function. For the spatial Fourier transform $F(k)$, a simple function involving a characteristic length scale $L$ of the problem was proposed. This function was constructed from some physical requirements and according to the modeling of a previous analytic calculation [31]. The separability assumption of Eq. (87) does not reproduce the features found in the simulations [52], so a time-dependent variable $L(t)$ was considered. The bubble radius $v\left(t-t_{*}\right)$ does not work either, and $L(t)$ is argued to represent the size of a typical colliding region, which initially grows but vanishes at the end of the transition. Therefore, $L(t)$ is modeled with

$$
L(t)=v \Delta t g(t)
$$

and the final form of $f$ is

$$
f(k, t)=L \sqrt{\Delta L}\left[\frac{1+\left(\frac{k L}{3}\right)^{2}}{1+\left(\frac{k L}{2}\right)^{2}+\left(\frac{k L}{3}\right)^{6}}\right]^{1 / 2} .
$$



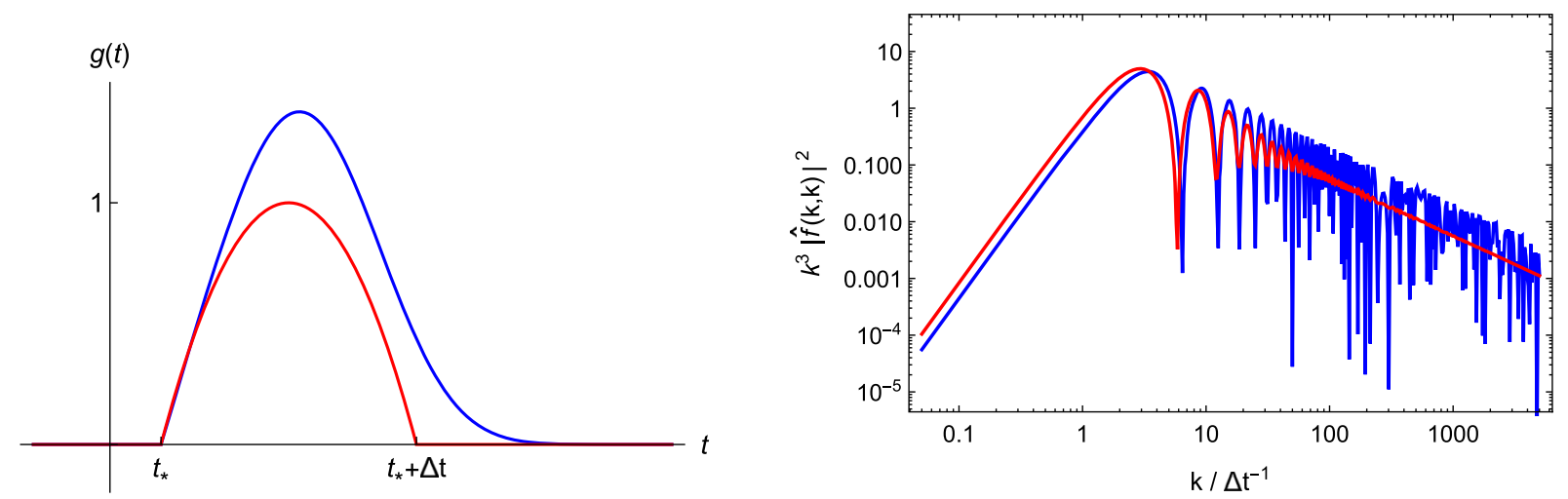

FIG. 20. Left: time evolution of the length scale of colliding walls $g(t)$ given by Eq. (91) (blue) and Eq. (93) (red). Right: the corresponding spectra with the approximation (89).

The global square root is motivated by the coherent-source approximation

$$
\Pi\left(t, t^{\prime}, k\right)=\sqrt{\Pi(t, t, k)} \sqrt{\Pi\left(t^{\prime}, t^{\prime}, k\right)}
$$

and the prefactor $\sqrt{L^{2} \Delta L}$ in Eq. (89) represents the volume of an uncollided wall, where $\Delta L$ parametrizes the wall thickness.

The result of the Fourier transform in Eq. (86) strongly depends on the continuity properties of the function $g(t)$, and the different functions considered in Ref. [51] give quite different behaviors of the GW spectrum. Based on results of computations of two bubble collisions from Ref. [27], it was argued that the time dependence is continuous but not differentiable. Indeed, using the simple function

$$
g(t)=\frac{4\left(t-t_{*}\right)\left[\Delta t-\left(t-t_{*}\right)\right]}{(\Delta t)^{2}}
$$

the construction (89) gives a GW spectrum of the required form. In particular, it reproduces the $k^{-1}$ decay for large frequencies found in simulations.

In our statistical treatment of the phase transition, the volume of uncollided walls is proportional to their uncollided area. Therefore, we may write the quantity $L^{2} \Delta L$ in the form $\langle S\rangle \Delta L$, and the size of the typical colliding region becomes

$$
L(t)=\sqrt{\langle S(t)\rangle} .
$$

The average area is given by Eq. (57), which roughly grows quadratically with the bubble size at the beginning of the phase transition and falls exponentially at the end. As we have seen, the behavior is qualitatively similar for an exponential nucleation rate and for a simultaneous nucleation. The latter is more consistent with the approximations of Eqs. (85), (88), and (89) (in particular, the assumptions of a constant $N / V$ and a single length scale $L$ ). Therefore, we shall consider this simpler case. Comparing Eq (92) with Eq. (88), we see that our definition of the function $g(t)$ should be ${ }^{19} g=\sqrt{\langle S\rangle} / d_{b}$, i.e.,

$$
g(t)=\sqrt{4 \pi}\left(\frac{t-t_{*}}{\Delta t}\right) \exp \left[-\frac{2 \pi}{3}\left(\frac{t-t_{*}}{\Delta t}\right)^{3}\right]
$$

for $t>t_{*}$, and $g(t)=0$ for $t<t_{*}$. In Fig. 20 we compare these functions and the corresponding form of GW spectra. These spectra have the same asymptotic behavior (namely, proportional to $k^{3}$ at low frequencies and to $k^{-1}$ at high frequencies). They have also a very similar shape near the peak, but they depart quantitatively for $k \gtrsim 10 \Delta t^{-1}$.

We remark that, in contrast to the quadratic function (91), our function (93) was derived from the average surface $\langle S\rangle$, which gives the actual "time window" inside which the source is active. This window will be present for any phenomenon originated at the bubble walls, such as GWs from corrugation instabilities. In order to investigate how the general features of the GW spectrum depend on this function and to what extent they depend on the spatial Fourier transform, let us set $F(k)=$ constant in Eq. (87). Thus, Eq. (89) becomes $f(k, t)=L(t) \sqrt{\Delta L}$, and we obtain a GW spectrum proportional to $k^{3}|\hat{g}(k)|^{2}$. The form of this spectrum is shown in Fig. 21, where we also consider the approximation (91) for $g(t)$. We see that the function $g(t)$ alone reproduces the general characteristics of the spectrum. Leaving aside the fact that this approximation gives only the schematic form of the spectrum, we notice that the definition of $g(t)$ from the average uncollided surface gives a more physical result. In particular, the quadratic approximation for $g(t)$ produces spurious oscillations in which the spectrum vanishes periodically.

The rough approximation we obtained by combining Eq. (92) with the previous approximations corresponds to

\footnotetext{
${ }^{19}$ As we have seen, for simultaneous nucleation we have $\Delta t \simeq t_{b}$ and $v \Delta t \simeq d_{b}$.
} 

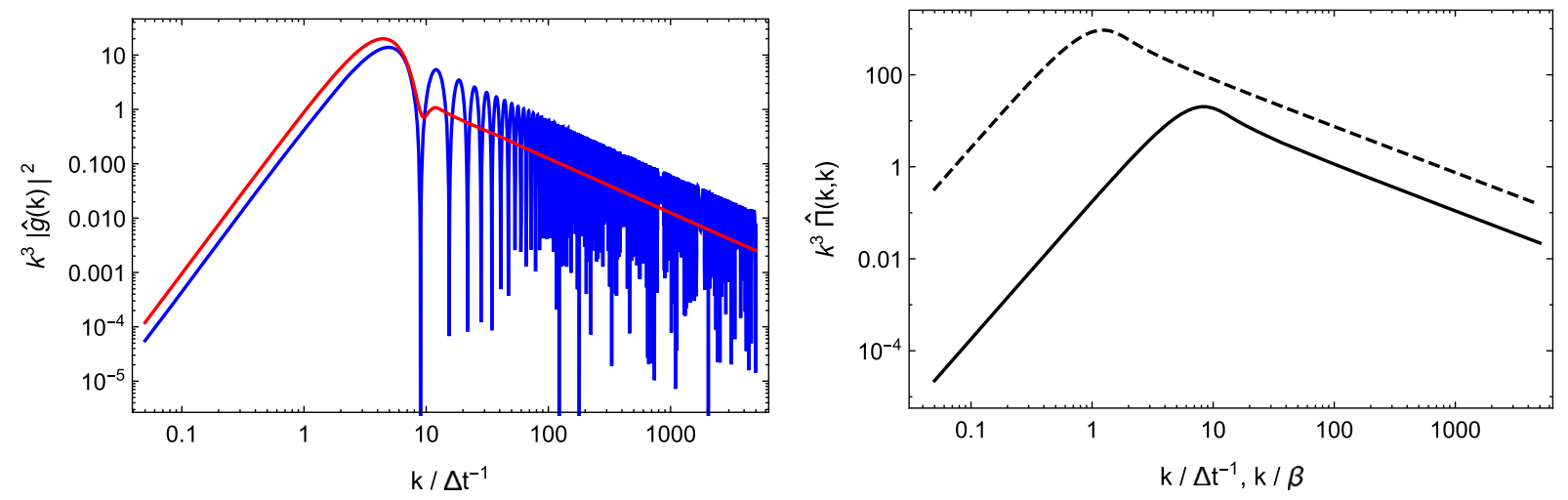

FIG. 21. Spectrum obtained without assuming a particular dependence on $k L$. Left: like in Fig. 20, but using the "window" function $g(t)$ alone. Right: assuming a source proportional to $\left\langle\Delta S(t) \Delta S\left(t^{\prime}\right)\right\rangle$, with time units $\Delta t$ (solid line) and $\beta^{-1}$ (dashed line).

using the coherent-source approximation (90), with an equal-time correlator proportional to $\langle S\rangle(t)$,

$$
\Pi\left(t, t^{\prime}\right)=\frac{2 \rho_{s}^{2} \Delta L N / V}{(2 \pi)^{3}} \sqrt{\frac{\langle S(t)\rangle\left\langle S\left(t^{\prime}\right)\right\rangle}{d_{b}^{4}}} .
$$

As already mentioned, it is to be expected that the relevant quantity is the correlation function ${ }^{20}\left\langle S(t) S\left(t^{\prime}\right)\right\rangle$ rather than $\langle S(t)\rangle\left\langle S\left(t^{\prime}\right)\right\rangle$. Nevertheless, these two quantities are not too different, as we have seen in the previous section (see Fig. 12). If we replace $\langle S(t)\rangle\left\langle S\left(t^{\prime}\right)\right\rangle$ with $\left\langle S(t) S\left(t^{\prime}\right)\right\rangle$, there is no actual reason for keeping the square root in Eq. (94), which was motivated by the coherent approximation. In any case, the square root does not change the qualitative features of the resulting spectrum, and a source of the form $\Pi\left(t, t^{\prime}\right) \propto\left\langle S(t) S\left(t^{\prime}\right)\right\rangle$ seems more reasonable. Moreover, these rough approximations do not take into account the transverse-traceless projection of the energy-momentum tensor, which, in particular, prevents a spherical bubble to radiate. The result should actually vanish in the uncorrelated case $\left\langle S(t) S\left(t^{\prime}\right)\right\rangle=\langle S(t)\rangle\left\langle S\left(t^{\prime}\right)\right\rangle$. To take this into account, we shall consider the approximation

$$
\Pi\left(t, t^{\prime}\right) \propto\left\langle\Delta S(t) \Delta S\left(t^{\prime}\right)\right\rangle .
$$

In the right panel of Fig. 21 we show the result of the Fourier transform for this model,

$$
\hat{\Pi}(k, k)=\int d t \int d t^{\prime} e^{i k\left(t-t^{\prime}\right)} \Pi\left(t, t^{\prime}\right) .
$$

The solid line corresponds to the same time units considered in the other figures, namely, the time interval $\Delta t$ in which the source is active. This curve has the same general characteristics as the others, but its shape is in agreement with more rigorous computations [32,52]. The dashed line

\footnotetext{
${ }^{20}$ In this approximation it makes no sense to consider the twobubble correlation function $\left\langle S(t) S^{\prime}\left(t^{\prime}\right)\right\rangle$.
}

is obtained by converting to the time unit $\beta^{-1}$ for an exponential nucleation rate, which for this model is rather artificial and is defined through the relation $\beta^{-1} \simeq \Delta t / 6.13$ (see Sec. IV B). We see that the peak of the spectrum is at $k / \beta \simeq 1$, in agreement with [32,52] (the amplitude of the spectrum does not have a physical meaning since we have omitted any proportionality constants).

\section{Topological defects}

The probability for different points on a bubble wall to be collided is a basic ingredient in the calculation of the dynamics of bubble intersections that enter the mechanism of defect trapping. This probability, which we have studied in previous sections for two points, is only the first step towards a calculation of defect formation using the statistical method considered in this paper. The probability of trapping a vortex, as explained in Sec. I, is related to that for three or more bubbles to get in contact with each other and enclose a region of false vacuum. Therefore, each of these bubbles must have at least two separate collided regions. It is clear that our method can be generalized to address this calculation. However, combining these probabilities with the condition that the overlapping bubbles form a closed chain does not seem trivial. Notice that the probability of forming a chain of several overlapped bubbles will also provide an analytic approach to the study of bubble percolation. We shall consider these applications elsewhere.

Nevertheless, from the two-point probabilities we can already see some differences between models which will be relevant for defect formation. An important element of the dynamics of defect formation is the time it takes a third bubble to arrive once two bubbles have collided. Therefore, the correlation between different parts of a bubble wall is very important. As we have seen in Sec. IV, for a simultaneous nucleation, the point correlation falls relatively quickly with the distance between the points, while for an exponentially growing rate all the points on the surface remain correlated. This implies that in the former 

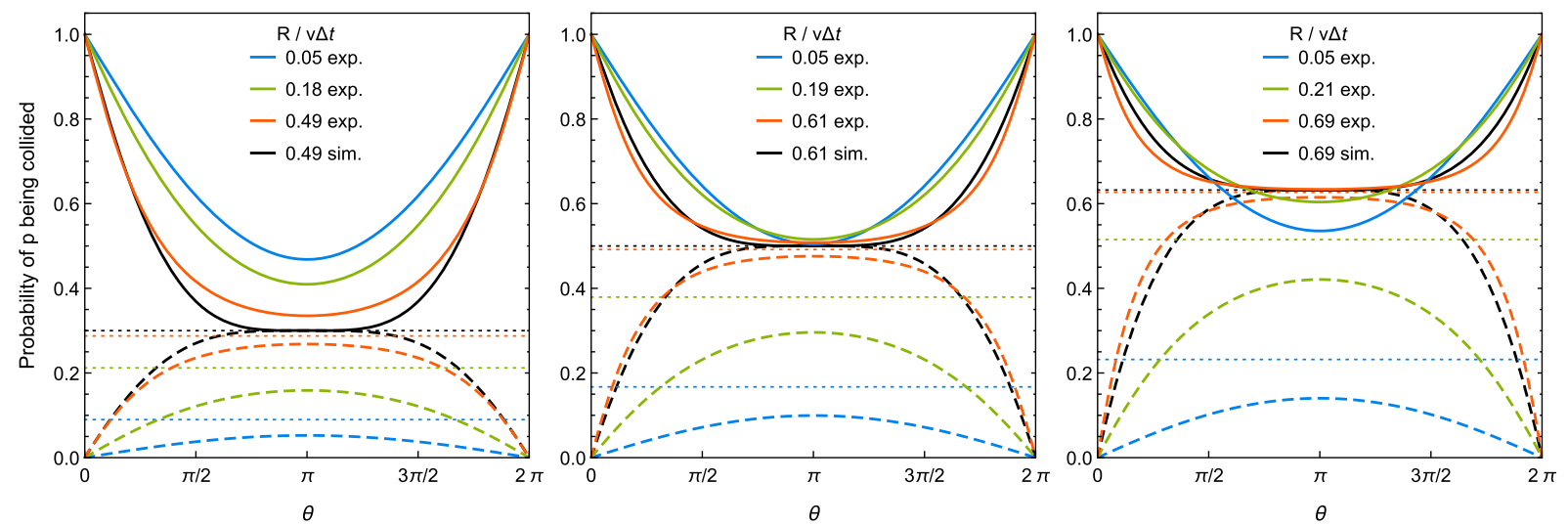

FIG. 22. Probability that $p$ is collided given that $p^{\prime}$ is collided (solid lines) and given that $p^{\prime}$ is not (dashed lines), when $f_{b}=0.3$ (left), $f_{b}=0.5$ (center), and $f_{b} \simeq 0.63$ (right). The horizontal dotted lines indicate the values of $1-P_{u}\left(t, t_{N}\right)$. The black line corresponds to the delta-function nucleation rate and the others to the exponential nucleation rate.

case it will be more probable that a given bubble at a given time has collided with more than one bubble. From the probabilities $P_{p \mid p^{\prime}}^{S}$ and $P_{p, p^{\prime}}^{S}$ derived in Sec. III, we may obtain two-point probabilities which are more directly related to such multiple collisions. For instance, the probability that a point $p$ is collided assuming that another point $p^{\prime}$ on the same bubble wall is collided is given by $\left(1-2 P_{u}+P_{p, p^{\prime}}^{S}\right) /\left(1-P_{u}\right)$, while the probability that $p$ is collided assuming that $p^{\prime}$ is not collided is given by $1-P_{p \mid p^{\prime}}^{S}$. The independent value of both probabilities is $1-P_{u}$.

In Fig. 22 we consider these probabilities for our two nucleation models. Each line corresponds to a given bubble radius and the probabilities are plotted as a function of the angle between the points. For a better comparison of the models, we use the unit of length $v \Delta t$ given by the duration of the phase transition, and the three panels show the situation at three representative moments corresponding to given values of the volume fraction. From left to right, we considered the time at which $f_{b}=0.3$ (this gives a rough estimation of the percolation time ${ }^{21}$ ), the time at which $f_{b}=0.5$, and the time at which $f_{b}=1-e^{-1}$. The black lines correspond to the simultaneous nucleation case, for which there is a single bubble size at a given time. For the case of the exponential rate, we consider three bubble sizes. One of them has the same value of the simultaneous case (orange lines), and the other two are smaller.

In the simultaneous case, the probability that a point is collided always approaches the independent value $f_{b}(t)$ for an angle $\theta \approx \pi / 2$. In the exponential case, for a bubble of the same radius the behavior is quite similar. However, for an exponential rate those bubbles are not relevant since smaller bubbles are much more abundant. The green lines correspond to the average bubble radius at each time and

\footnotetext{
${ }^{21}$ Simulations with overlapping spheres of equal radius give $I \simeq 0.34$ and $f_{b} \simeq 0.29$ (see, e.g., [53]).
}

the blue lines correspond to an even smaller bubble. For these bubbles the behavior is quite different. In the first place, the general probability that a point $p$ on the bubble wall is collided (dotted lines) decreases from the value $f_{b}$ corresponding to an arbitrary point in space (black dotted line). If we further assume that another point $p^{\prime}$ on the wall is uncollided, the probability that $p$ is collided is even smaller (dashed lines). The separation between dotted and dashed lines indicates that the probabilities for the two points are never independent.

On the other hand, if we assume that a point $p^{\prime}$ on the wall is already collided, the probability that $p$ is collided is higher (solid lines). For $\theta=0$ this probability is 1 , while for $\theta=\pi$ it gets more or less close to the value $f_{b}$, depending on the bubble size and the time. However, it is important to remark that this is not the independent value for a point on the surface but for an arbitrary point in space. For small bubbles, the solid lines are far from the dotted lines, which means that the probability of $p$ being collided is quite higher than the independent value if $p^{\prime}$ is collided. Therefore, under this condition, $p$ has a probability $\sim f_{b}$ of being collided, but it is likely that $p$ has collided with the same bubble as $p^{\prime}$. In contrast, for the simultaneous case the solid curve rapidly approaches the independent value, indicating that we have a probability $f_{b}$ that the point has collided with any bubble. In this scenario, simultaneous multiple collisions, which favor defect trapping, are more probable.

\section{CONCLUSIONS}

We have studied bubble wall correlations in cosmological phase transitions using a statistical treatment of the bubble kinematics. Specifically, we have calculated conditional and joint probabilities for a set of arbitrary points of space to remain in the false vacuum at different times, conditional and joint probabilities for two points on a bubble wall or on two different bubble walls to remain 
uncollided, and the probability that a point on a bubble wall is uncollided, in the presence of another bubble. We have used these probabilities to study the evolution of the envelope of uncollided walls as well as spacial and temporal correlations within this surface. Our general results depend on the nucleation rate $\Gamma(t)$ and the wall velocity $v(t)$, and can thus be applied to different types of phase transitions. We have considered a few specific models and discussed the application of our results to the calculation of possible remnants of the phase transition, such as the baryon asymmetry of the Universe, a stochastic background of gravitational waves, and topological defects.

Our general treatment of probabilities is based on the two basic ingredients $\Gamma(t)$ and $v(t)$, which are assumed to depend only on time. Although this is a widely used simplification, it is not always valid. As we have mentioned, this approximation is valid for detonations (which are supersonic) or for deflagrations with velocities $v \lesssim 0.1$. In the intermediate case of deflagrations which are not very slow $(0.1 \lesssim v \lesssim 0.6)$, a shock wave moving slightly faster than the speed of sound reheats the plasma in front of the wall. In this case, the kinematic treatment can still be simplified by taking into account the fact that, in a region of radius $R_{\mathrm{sh}} \approx\left(c_{s} / v\right) R$ around each bubble, the nucleation rate vanishes [54]. This approximation assumes that the reheating caused by a single bubble is enough to turn off the nucleation rate, so the inhomogeneous temperature resulting from several shock waves is irrelevant. However, some approximation is still required for the velocity, which is not as sensitive to temperature. Our results can in principle be adapted to such a treatment.

Some generalizations of our derivations are straightforward, such as considering more than two points on bubble walls and calculating the probability that some of them are collided and some others are not. However, further development would be necessary in order to address certain applications such as the study of percolation or a thorough calculation topological defect formation, which require considering the dynamics of multiple bubble collisions. Here we have focused on two-point probabilities, and we have used these probabilities to discuss surface correlations between different bubbles, between a single bubble at different times, and between different parts of a bubble wall at a given time. These quantities are relevant for the consequences of the transition, and we have discussed a few examples.

In the first place, we have discussed the importance of the total uncollided wall area $\left\langle S_{\text {tot }}\right\rangle$ as a weight for baryogenesis in an electroweak phase transition with a varying wall velocity. Indeed, a small velocity is favorable for baryogenesis, and in this case the velocity varies due to reheating during the transition. We have considered adequate analytic approximations for the wall velocity for this case, and we have seen that, depending on the model, the uncollided wall area will amplify the possible enhancement or suppression of the baryon asymmetry due to the velocity variation.

We have argued that the uncollided wall area plays a relevant role in the generation of gravitational waves from bubble walls. This includes, e.g., GW generation from corrugation instabilities, but is especially important for the bubble collision mechanism. We have analyzed the role of the quantity $\langle S(t)\rangle$ as a time window for the source of gravitational waves and the dependence of the GW spectrum on the correlation function $\left\langle S(t) S\left(t^{\prime}\right)\right\rangle$. In particular, we have shown that the latter, without further considerations on the spatial dependence of the source, reproduces the correct shape of the GW spectrum, the peak frequency, and the asymptotic behavior at low and high frequencies. We have seen that the qualitative behavior of surface correlations is similar for two very different models of bubble nucleation, namely, a delta-function rate and an exponential rate. Therefore, we expect the general characteristics of the spectrum to be similar for different models. This conclusion is supported by specific calculations [55].

In contrast, we have argued that the spatial correlation on a bubble wall is particularly relevant for topological defect formation, and this correlation may behave very differently depending on the characteristics of bubble nucleation. For instance, for an exponential nucleation rate, we have a continuous nucleation of very small bubbles. These small bubbles nucleate in the false vacuum regions between larger bubbles and are captured by the latter before they can collide with each other. In contrast, if all the bubbles nucleate simultaneously, the collisions occur between bubbles of the same size, and we expect that in this case it will be easier to enclose a false vacuum region. We have reinforced this argument by analyzing the probability for two points on a bubble wall to be collided.

\section{ACKNOWLEDGMENTS}

This work was supported by CONICET Grant No. PIP 11220130100172 and Universidad Nacional de Mar del Plata, Grant No. EXA897/18.

\section{APPENDIX: BUBBLE CONFIGURATIONS FOR TWO-POINT CONDITIONAL PROBABILITY}

In this Appendix we consider the two orderings of the times $t_{N}, t_{N}^{\prime}, t^{\prime}$, which were not considered in Fig. 7.

The case $t_{N}^{\prime}<t_{N}<t^{\prime}$ is illustrated in Fig. 23 for some values of $t^{\prime \prime}$. The configuration for $t^{\prime \prime}>t^{\prime}$ is not shown, since in this case the nucleation at $t^{\prime \prime}$ cannot affect the events at times $t^{\prime}, t_{N}^{\prime}$, or $t_{N}$, and we have

$$
d P\left(t^{\prime \prime}\right)=d t^{\prime \prime} \Gamma\left(t^{\prime \prime}\right) \frac{4 \pi}{3} R\left(t^{\prime \prime}, t\right)^{3}, \quad\left(t^{\prime \prime}>t^{\prime}\right) .
$$

In the case $t_{N}<t^{\prime \prime}<t^{\prime}$ (Fig. 23, left panel), a bubble nucleated at time $t^{\prime \prime}$ may have eaten the point $p^{\prime}$ at time $t^{\prime}$, so we must exclude the striped region, 

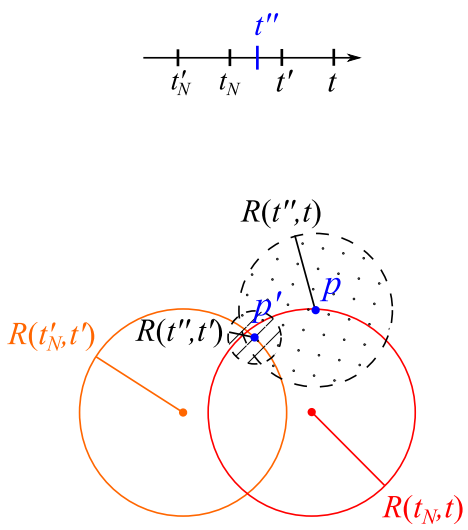
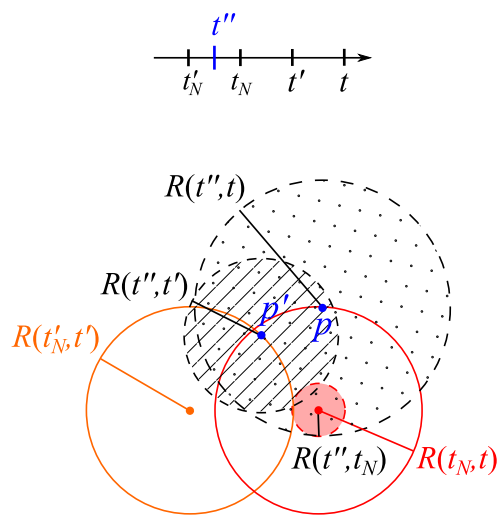
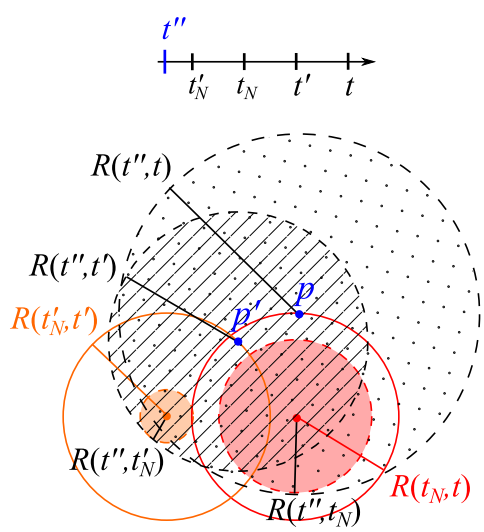

FIG. 23. Like Fig. 7, for the time ordering $t_{N}^{\prime}<t_{N}<t^{\prime}$.

$$
d P\left(t^{\prime \prime}\right)=d t^{\prime \prime} \Gamma\left(t^{\prime \prime}\right)\left[\frac{4 \pi}{3} R\left(t^{\prime \prime}, t\right)^{3}-V_{\cap}\right] \quad\left(t_{N}^{\prime}<t^{\prime \prime}<t^{\prime}\right) .
$$

For $t_{N}^{\prime}<t^{\prime \prime}<t_{N}$ (Fig. 23, central panel), the nucleation at $t^{\prime \prime}$ may also prevent the nucleation of bubble $B$. Therefore, we must exclude the pink region as well as the striped region, and we have

$$
\begin{aligned}
d P\left(t^{\prime \prime}\right)= & d t^{\prime \prime} \Gamma\left(t^{\prime \prime}\right)\left[\frac{4 \pi}{3} R\left(t^{\prime \prime}, t\right)^{3}-\frac{4 \pi}{3} R\left(t^{\prime \prime}, t_{N}\right)^{3}-V_{\cap}+V_{\cap}^{\prime}\right], \\
& \left(t_{N}^{\prime}<t^{\prime \prime}<t_{N}\right) .
\end{aligned}
$$

Finally, for $t^{\prime \prime}<t_{N}^{\prime}$ (Fig. 23, right panel), the nucleation at $t^{\prime \prime}$ may also prevent the nucleation of bubble $B^{\prime}$, but the region which can affect this event (orange shade) is completely contained within the striped region, and we obtain again Eq. (A3).

Now let us consider the case $t_{N}^{\prime}<t^{\prime}<t_{N}$, which is illustrated in Fig. 24. For $t^{\prime \prime}>t^{\prime}$, the bubble $B^{\prime}$ and the point $p^{\prime}$ cannot be affected by nucleations at $t^{\prime \prime}$, and we have, for the case $t^{\prime \prime}>t_{N}$ (not shown in the figure)

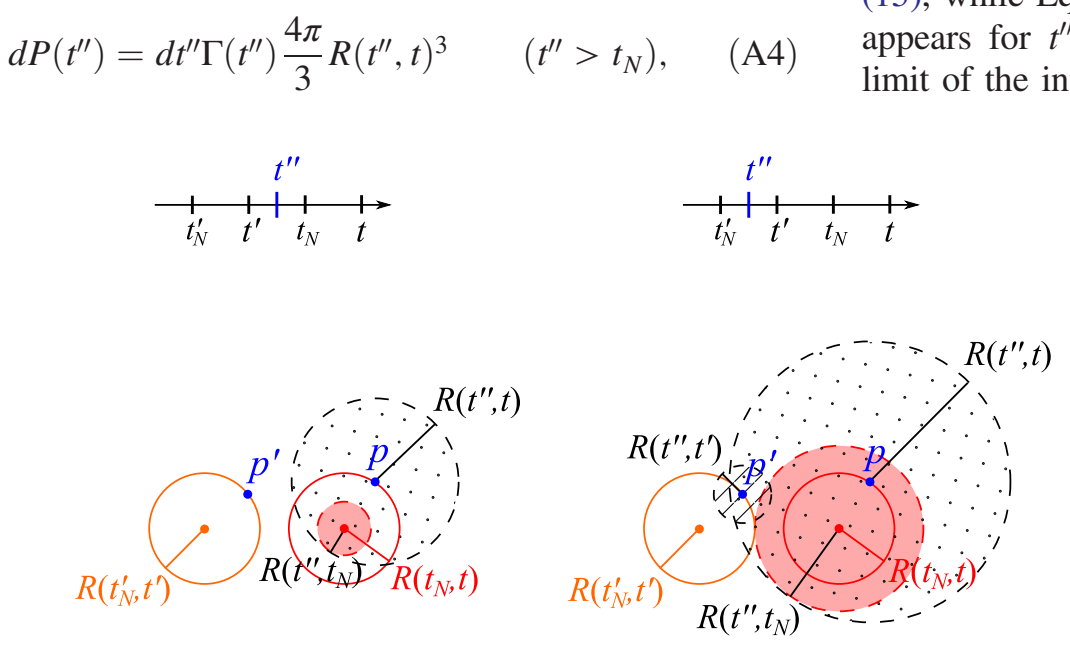

while for the case $t^{\prime \prime}<t_{N}$ (Fig. 24, left panel),

$d P\left(t^{\prime \prime}\right)=d t^{\prime \prime} \Gamma\left(t^{\prime \prime}\right) \frac{4 \pi}{3}\left[R\left(t^{\prime \prime}, t\right)^{3}-R\left(t^{\prime \prime}, t_{N}\right)^{3}\right] \quad\left(t^{\prime}<t^{\prime \prime}<t_{N}\right)$.

For $t_{N}^{\prime}<t^{\prime \prime}<t^{\prime}$ (Fig. 24, central panel), the point $p^{\prime}$ can be affected, and we have

$$
\begin{aligned}
d P\left(t^{\prime \prime}\right)= & d t^{\prime \prime} \Gamma\left(t^{\prime \prime}\right)\left[\frac{4 \pi}{3} R\left(t^{\prime \prime}, t\right)^{3}-\frac{4 \pi}{3} R\left(t^{\prime \prime}, t_{N}\right)^{3}\right. \\
& \left.-V_{\cap}+V_{\cap}^{\prime}\right] \quad\left(t_{N}^{\prime}<t^{\prime \prime}<t^{\prime}\right) .
\end{aligned}
$$

Finally, for $t^{\prime \prime}<t_{N}^{\prime}$ (Fig. 24, right panel), the nucleation of $B^{\prime}$ can also be affected, but this is already taken into account in Eq. (A6), since the orange region is always contained in the striped region.

These results lead to Eqs. (42)-(43). In particular, Eqs. (A2) and (A6) show that the volume $V_{\cap}$ appears for $t^{\prime \prime}<t^{\prime}$, as expressed by the upper limit of the integral (15), while Eqs. (A3) and (A6) show that the volume $V_{\cap}^{\prime}$ appears for $t^{\prime \prime}<\min \left\{t_{N}, t^{\prime}\right\}$, as expressed by the upper limit of the integral (43).

FIG. 24. Like Fig. 7, for the time ordering $t_{N}^{\prime}<t^{\prime}<t_{N}$. 
[1] M. S. Turner, E. J. Weinberg, and L. M. Widrow, Bubble nucleation in first order inflation and other cosmological phase transitions, Phys. Rev. D 46, 2384 (1992).

[2] M. Kamionkowski, A. thur Kosowsky, and M. S. Turner, Gravitational radiation from first order phase transitions, Phys. Rev. D 49, 2837 (1994).

[3] S. R. Coleman, The fate of the false vacuum. 1. Semiclassical theory, Phys. Rev. D 15, 2929 (1977); Erratum, Phys. Rev. D 16, 1248 (1977).

[4] C. G. Callan, Jr. and S. R. Coleman, The fate of the false vacuum. 2. First quantum corrections, Phys. Rev. D 16, 1762 (1977).

[5] D. Bodeker and G. D. Moore, Can electroweak bubble walls run away?, J. Cosmol. Astropart. Phys. 05 (2009) 009.

[6] D. Bodeker and G. D. Moore, Electroweak bubble wall speed limit, J. Cosmol. Astropart. Phys. 05 (2017) 025.

[7] S. Hche, J. Kozaczuk, A. J. Long, J. Turner, and Y. Wang, Towards an all-orders calculation of the electroweak bubble wall velocity, arXiv:2007.10343v2.

[8] A. D. Linde, Fate of the false vacuum at finite temperature: Theory and applications, Phys. Lett. 100B, 37 (1981).

[9] A. D. Linde, Decay of the false vacuum at finite temperature, Nucl. Phys. B216, 421 (1983); Erratum, Nucl. Phys. B223, 544 (1983).

[10] N. Turok, Electroweak Bubbles: Nucleation and Growth, Phys. Rev. Lett. 68, 1803 (1992).

[11] G. D. Moore and T. Prokopec, How fast can the wall move? A study of the electroweak phase transition dynamics, Phys. Rev. D 52, 7182 (1995).

[12] E. Witten, Cosmic separation of phases, Phys. Rev. D 30, 272 (1984).

[13] M. Laine, Bubble growth as a detonation, Phys. Rev. D 49, 3847 (1994).

[14] J. Ignatius, K. Kajantie, H. Kurki-Suonio, and M. Laine, The growth of bubbles in cosmological phase transitions, Phys. Rev. D 49, 3854 (1994).

[15] A. Megevand and S. Ramirez, Bubble nucleation and growth in very strong cosmological phase transitions, Nucl. Phys. B919, 74 (2017).

[16] J. Ellis, M. Lewicki, and J. M. No, On the maximal strength of a first-order electroweak phase transition and its gravitational wave signal, J. Cosmol. Astropart. Phys. 04 (2019) 003.

[17] R. Jinno, T. Konstandin, and M. Takimoto, Relativistic bubble collisions-A closer look, J. Cosmol. Astropart. Phys. 09 (2019) 035.

[18] V. A. Kuzmin, V. A. Rubakov, and M. E. Shaposhnikov, On the anomalous electroweak baryon number nonconservation in the Early Universe, Phys. Lett. B 155, 36 (1985).

[19] A. G. Cohen, D. B. Kaplan, and A. E. Nelson, Progress in electroweak baryogenesis, Annu. Rev. Nucl. Part. Sci. 43, 27 (1993).

[20] A. Riotto and M. Trodden, Recent progress in baryogenesis, Annu. Rev. Nucl. Part. Sci. 49, 35 (1999).

[21] D. E. Morrissey and M. J. Ramsey-Musolf, Electroweak baryogenesis, New J. Phys. 14, 125003 (2012).

[22] M. S. Turner and F. Wilczek, Relic Gravitational Waves and Extended Inflation, Phys. Rev. Lett. 65, 3080 (1990).
[23] C. Caprini et al., Science with the space-based interferometer eLISA. II: Gravitational waves from cosmological phase transitions, J. Cosmol. Astropart. Phys. 04 (2016) 001.

[24] C. Caprini et al., Detecting gravitational waves from cosmological phase transitions with LISA: An update, J. Cosmol. Astropart. Phys. 03 (2020) 024.

[25] A. Kosowsky, M. S. Turner, and R. Watkins, Gravitational radiation from colliding vacuum bubbles, Phys. Rev. D 45, 4514 (1992).

[26] M. Hindmarsh, S. J. Huber, K. Rummukainen, and D. J. Weir, Gravitational Waves from the Sound of a First Order Phase Transition, Phys. Rev. Lett. 112, 041301 (2014).

[27] A. Kosowsky and M. S. Turner, Gravitational radiation from colliding vacuum bubbles: Envelope approximation to many bubble collisions, Phys. Rev. D 47, 4372 (1993).

[28] T. W. B. Kibble, Topology of cosmic domains and strings, J. Phys. A 9, 1387 (1976).

[29] T. W. B. Kibble and A. Vilenkin, Phase equilibration in bubble collisions, Phys. Rev. D 52, 679 (1995).

[30] J. Borrill, T. W. B. Kibble, T. Vachaspati, and A. Vilenkin, Defect production in slow first order phase transitions, Phys. Rev. D 52, 1934 (1995).

[31] C. Caprini, R. Durrer, and G. Servant, Gravitational wave generation from bubble collisions in first-order phase transitions: An analytic approach, Phys. Rev. D 77, 124015 (2008).

[32] R. Jinno and M. Takimoto, Gravitational waves from bubble collisions: An analytic derivation, Phys. Rev. D 95, 024009 (2017).

[33] P. J. Steinhardt, Relativistic detonation waves and bubble growth in false vacuum decay, Phys. Rev. D 25, 2074 (1982).

[34] M. Gyulassy, K. Kajantie, H. Kurki-Suonio, and L. D. McLerran, Deflagrations and detonations as a mechanism of hadron bubble growth in supercooled quark gluon plasma, Nucl. Phys. B237, 477 (1984).

[35] H. Kurki-Suonio, Deflagration Bubbles in the quarkhadron phase transition, Nucl. Phys. B255, 231 (1985).

[36] H. Kurki-Suonio and M. Laine, Supersonic deflagrations in cosmological phase transitions, Phys. Rev. D 51, 5431 (1995).

[37] K. Enqvist, J. Ignatius, K. Kajantie, and K. Rummukainen, Nucleation and bubble growth in a first order cosmological electroweak phase transition, Phys. Rev. D 45, 3415 (1992).

[38] A. F. Heckler, The effects of electroweak phase transition dynamics on baryogenesis and primordial nucleosynthesis, Phys. Rev. D 51, 405 (1995).

[39] A.H. Guth and S.H.H. Tye, Phase Transitions and Magnetic Monopole Production in the Very Early Universe, Phys. Rev. Lett. 44, 631 (1980); Erratum, Phys. Rev. Lett. 44, 963 (1980).

[40] A. Megevand and F. Astorga, Generation of baryon inhomogeneities in the electroweak phase transition, Phys. Rev. D 71, 023502 (2005).

[41] M. Hindmarsh, S. J. Huber, K. Rummukainen, and D. J. Weir, Numerical simulations of acoustically generated gravitational waves at a first order phase transition, Phys. Rev. D 92, 123009 (2015). 
[42] J. M. Cline and K. Kainulainen, Electroweak baryogenesis at high bubble wall velocities, Phys. Rev. D 101, 063525 (2020).

[43] A. Megevand, Effect of reheating on electroweak baryogenesis, Phys. Rev. D 64, 027303 (2001).

[44] A. Megevand, First order cosmological phase transitions in the radiation dominated era, Phys. Rev. D 69, 103521 (2004).

[45] A. Megevand and A. D. Sanchez, Supercooling and phase coexistence in cosmological phase transitions, Phys. Rev. D 77, 063519 (2008).

[46] A. Mégevand and S. Ramírez, Bubble nucleation and growth in slow cosmological phase transitions, Nucl. Phys. B928, 38 (2018).

[47] B. Link, Deflagration Instability in the Quark-Hadron Phase Transition, Phys. Rev. Lett. 68, 2425 (1992).

[48] P. Y. Huet, K. Kajantie, R. G. Leigh, B.-H. Liu, and L. D. McLerran, Hydrodynamic stability analysis of burning bubbles in electroweak theory and in QCD, Phys. Rev. D 48, 2477 (1993).

[49] A. Megevand and F. A. Membiela, Stability of cosmological deflagration fronts, Phys. Rev. D 89, 103507 (2014).
[50] A. Megevand, F. A. Membiela, and A. D. Sanchez, Lower bound on the electroweak wall velocity from hydrodynamic instability, J. Cosmol. Astropart. Phys. 03 (2015) 051.

[51] C. Caprini, R. Durrer, T. Konstandin, and G. Servant, General properties of the gravitational wave spectrum from phase transitions, Phys. Rev. D 79, 083519 (2009).

[52] S. J. Huber and T. Konstandin, Gravitational wave production by collisions: More bubbles, J. Cosmol. Astropart. Phys. 09 (2008) 022.

[53] M. D. Rintoul and S. Torquato, Precise determination of the critical threshold and exponents in a threedimensional continuum percolation model, J. Phys. A 30, L585 (1997).

[54] L. Leitao, A. Megevand, and A. D. Sanchez, Gravitational waves from the electroweak phase transition, J. Cosmol. Astropart. Phys. 10 (2012) 024.

[55] R. Jinno, S. Lee, H. Seong, and M. Takimoto, Gravitational waves from first-order phase transitions: Towards model separation by bubble nucleation rate, J. Cosmol. Astropart. Phys. 11 (2017) 050. 